\title{
Nonlinear dynamic behavior of a flexible structure to combined external acoustic and parametric excitation
}

\author{
Paulo S. Varoto and Demian G. Silva \\ Mechanical Engineering Department, School of Engineering of Sao Carlos, University of Sao Paulo, Av. \\ Trabalhador Saocarlense 400, Sao Carlos, SP 13566-590, Brazil \\ E-mail:varoto@sc.usp.br
}

\begin{abstract}
Flexible structures are frequently subjected to multiple inputs when in the field environment. The accurate determination of the system dynamic response to multiple inputs depends on how much information is available from the excitation sources that act on the system under study. Detailed information include, but are not restricted to appropriate characterization of the excitation sources in terms of their variation in time and in space for the case of distributed loads. Another important aspect related to the excitation sources is how inputs of different nature contribute to the measured dynamic response. A particular and important driving mechanism that can occur in practical situations is the parametric resonance. Another important input that occurs frequently in practice is related to acoustic pressure distributions that is a distributed type of loading. In this paper, detailed theoretical and experimental investigations on the dynamic response of a flexible cantilever beam carrying a tip mass to simultaneously applied external acoustic and parametric excitation signals have been performed. A mathematical model for transverse nonlinear vibration is obtained by employing Lagrange's equations where important nonlinear effects such as the beam's curvature and quadratic viscous damping are accounted for in the equation of motion. The beam is driven by two excitation sources, a sinusoidal motion applied to the beam's fixed end and parallel to its longitudinal axis and a distributed sinusoidal acoustic load applied orthogonally to the beam's longitudinal axis. The major goal here is to investigate theoretically as well as experimentally the dynamic behavior of the beam-lumped mass system under the action of these two excitation sources. Results from an extensive experimental work show how these two excitation sources interacts for various testing conditions. These experimental results are validated through numerically simulated results obtained from the solution of the system's nonlinear equation of motion.
\end{abstract}

\section{Introduction}

In the field environment, a given structure may be subjected to excitations of different nature. This is typically the case of flight hardware, a satellite for example. During the flight, the satellite is subjected to contact excitations arising from the connections with the launch vehicle and possibly acoustic excitations due to internal noise generation mechanisms. A CD player mounted on the dash board of an automobile is another good example of a system that may suffer from multiple excitations. In both cases, the structure needs to survive to all forms of excitations and still operate accordingly, and obviously, this is a major concern of design and test personnel involved with each particular product.

From the modelling viewpoint, if it is assumed that the structure under investigation presents a linear dynamic behavior, governed by infinitesimal vibration amplitudes, then the analytical description of the structure is made through a set of nonhomogeneous linear ordinary differential equations with constant coefficients. This is typically the description obtained when the classic finite element method is used. In this case, all the excitations, forces and moments appear on the right hand side of the equations of motion. However, in the event of high excitation amplitudes associated with higher flexibility presented by nowadays structural assemblies the system may present nonlinear vibration behavior, and the assumption of linearity fails to adequately describe its dynamic behavior. 
In practice, all structures are essentially nonlinear, and in this case the mathematical models used to describe the dynamics of the system are considerably more complex than the models used in the classical linear theory. The type of the nonlinearity present, arising for example from geometry or material property will reflect on differences exhibited by the equations of motion used to model the system's dynamic behavior. Particularly, one of these differences occurs in the presence of time varying coefficients on the left hand side of the equation of motion, frequently in the term that describes the elastic forces. This term constitutes the parametric excitation [4,14], and due to this excitation mechanism, the structure may present a special and very important vibration condition, characterized by large amplitudes and that is called parametric resonance.

Faraday [6] in 1883 was probably one of the first to observe the parametric resonance phenomenon by noticing that the wine in glass presented a strong oscillation at a frequency approximately half of the exciting frequency provided by the movement of the finger around the edge of the glass. Only in 1887, Lord Rayleigh [6] provided a mathematical explanation the phenomenon previously observed by Faraday which was named parametric resonance [4,6,14].

Beliav, 1924 [6], apparently was the first in providing an analysis of the parametric resonance for a purely structural system that consisted a straight elastic column pinned at both ends and subjected to an axial periodic sinusoidal force at a constant excitation frequency. The results shown that the column could vibrate with half of the original excitation frequency if this frequency was close to a bending natural frequency of the column, even if the amplitude of the applied force was kept below of the column buckling load [6]. After Beliav, some other authors have analyzed the parametric resonance in other kinds of structural elements. Einaudi in 1936 was the first to study this phenomenon in plates and Bublik and Merkulov in 1942 were the pioneers investigating the problem in cylindrical shells [6]. Since the early investigations on the parametric resonance in purely structural systems, the scientific community have dedicated great efforts in the study of beam type structural elements.

Zavodney and Nayfeh [18] in 1989, performed an extensive theoretical and experimental work in three different slender cantilever beams carrying a lumped mass, two steel and one composite graphite-epoxy beam. In all situations the beams were vertically supported and subjected to a principal parametric base excitation. The slender beam Euler-Bernoulli has been used to derive the governing non-linear partial differential equation and the Galerkin's method was used to obtain the discrete equation of motion. The method of multiple scales was employed to determine an approximate solution for the time domain equation of motion and experiments were carried out on the metallic beams and later on the composite beam. All of the metallic beams has failed prematurely due to the very large response amplitudes caused by the parametric resonance phenomenon.

Cartmell $[5,8]$ in 1990, showed an important work on the derivation of the equation of motion applied to a cantilever beam subjected to base excitation. A similar configuration to that used by Zavodney and Nayfeh [18] was employed except that in this case, the direction of the excitation was in the transverse direction instead of vertical. Initially, the kinematics of the problem was established and subsequently the Lagrange's formulation was applied to find the equation of motion. The main goal in Cartmell's work was to illustrate the use of classical engineering theories in the accurate modelling of a very simple structure, and to highlight the conceptualization of a three-dimensional problem.

Anderson et al. [1] have performed an experimental and theoretical investigation into the first and second mode responses of a parametrically excited slender cantilever beam without added mass. It has been shown that the inclusion of a quadratic damping in the analytical model significantly improves the agreement between the experimental and theoretical results. In addition, the experimental results obtained verified that the often ignored non-linear curvature terms play a crucial role in the response of the first mode and that the non-linear inertia terms also plays an important role in the response of the second mode.

HaQuang et al. [11] in 1987 performed an important analytical investigation on a weakly quadratic and cubic nonlinear multiple degrees of freedom system including both external and parametric excitations. They used the multiple scales method to investigate the steady state responses when the frequency of the parametric excitation is near a natural frequency of the system for three conditions: (i) no external excitation present; (ii) external excitation present but not involved in the resonance condition; and (iii) external excitation frequency equals the parametric excitation frequency. They used frequency-response curves to illustrate the effects of damping, excitation amplitudes, and a phase difference between the parametric and external excitations. They affirmed yet that the most surprising result was the existence of stable, multi-modal, steady state responses.

HaQuang et al. [10] still in 1987 published another important and extensive theoretical study focused on the interaction between parametric and external excitations acting on a weakly quadratic and cubic nonlinear system. 
Essentially the same theoretical approach used in the previous work was employed here to analyze the system's dynamic response to the combined excitations. The authors pointed here to the difficulty in defining suitable control strategies when the combined excitations act on the test structure.

Up to date, only a few experimental investigations on the dynamic behavior of a structural system subject to a combined parametric and external excitation have been performed, probably due the complexity and intrinsic problems related with the tests. Among these, Nayfeh et al. [15] in 1995, also cited in the recently published text book [13] studied a taut string made of latex with an inner diameter of 1/8" and outer diameter of 1/4" and 2.13 m of span. An electrodynamic vibration exciter was used to supply an oscillatory boundary condition at one end of the string, and the other end was fixed at a rigid wall. The axis of the shaker was inclined so that the motion had components both parallel and transverse to the axis of the string. The motion of the exciter's armature was monitored by an accelerometer mounted to the shaker head and the displacement of the string was measured by an optical system mounted close to the fixed end of the string. Several experimental frequency-response curves were obtained for various physical parameters of the system and compared with the theoretical results.

Among the external excitation mechanisms that could act on the structure in the field, acoustic loadings play an important role in several practical situations as for example in the study of fatigue on aircraft surfaces due to acoustic pressure waves generated by the engines [3]. From the laboratory viewpoint, the problem of performing tests in a flexible structure under the combined effect of a parametric and external acoustic excitation is indeed a challenging problem. Based on this argument, the major goal of this paper is to perform a detailed theoretical and experimental investigation on the dynamic response properties of a slender beam-lumped mass system undergoing combined and simultaneous parametric and external acoustic excitations. A nonlinear mathematical model is derived based on energy quantities. Some fundamental preliminary tests are performed in order to obtain the modal properties of the structure and the characterization of the excitation sources. In the sequence, an extensive nonlinear experimental analysis is performed in various testing conditions by simultaneously applying the structural and acoustic excitations to the structure. The system's response is measured and compared with numerically obtained results from the system's model.

\section{Theoretical approach}

To investigate the effects of the combined excitations from the analytical viewpoint, the structure shown in Fig. 1 was built. It is composed of a slender stainless steel ASTM A240 beam, with dimensions of $100 \mathrm{~mm}$ in length, $20 \mathrm{~mm}$ in width and $1 \mathrm{~mm}$ in thickness. The lumped mass is composed of carbon steel ASTM A36, with dimensions of $10 \mathrm{~mm}$ in length, $40 \mathrm{~mm}$ in width and $20 \mathrm{~mm}$ in height. The opposite beam's end is clamped to a rigid base built from carbon ASTM A36 steel.

This structure will be simultaneously submitted to two external driving mechanisms, a parametric excitation due to a base excitation applied by an electrodynamic shaker and a transverse acoustic external excitation applied by an acoustic source positioned parallel to the structure. The following assumptions are made in deriving the equation for the beam's transverse motion:

H1 The beam's transverse vibration is purely planar and completely described in the $O Z X$ plane since the lumped mass is symmetric with respect to the centerline and the beam is kept short (less than thirty times the beam's width) then the torsional modes can be neglected in the analysis.

H2 The thickness of the beam is small compared to the length so that the effects of shear strain and rotatory inertia of the beam can be neglected.

H3 All the transverse sections to the centerline of the beam remains plane and normal to the centerline.

H4 It is assumed that the beam's material obeys the constitutive Hook's law, is isotropic and there is neither plastic strain or internal heat generated during the vibration.

H5 The mass of the beam when compared with the lumped mass is very small, then its effect can be neglected and the system will contain a single degree of freedom.

H6 Potential fluid-structure interactions between the acoustic media and the structure under study are neglected, particularly in terms of additional damping added to the system due to the fluid motion caused by the acoustic excitation source. 


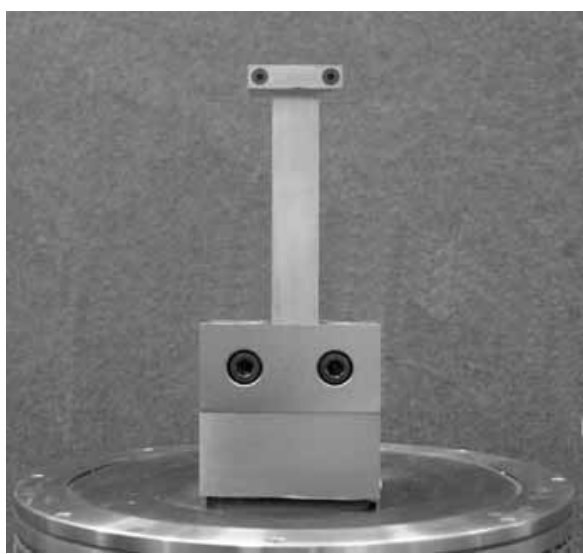

(a)

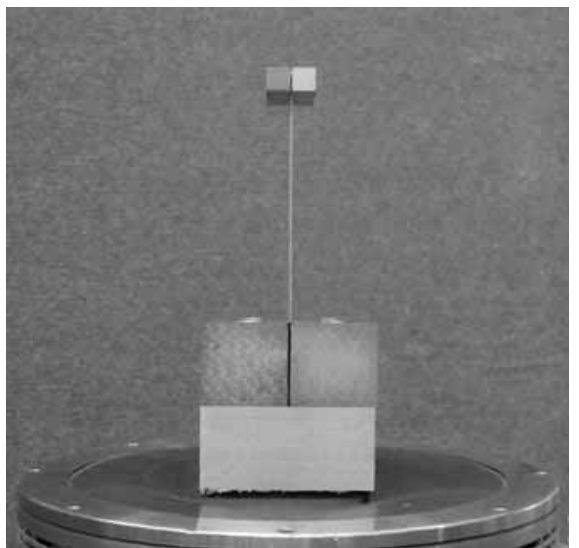

(b)

Fig. 1. Physical system under investigation: (a) frontal view; (b) lateral view.

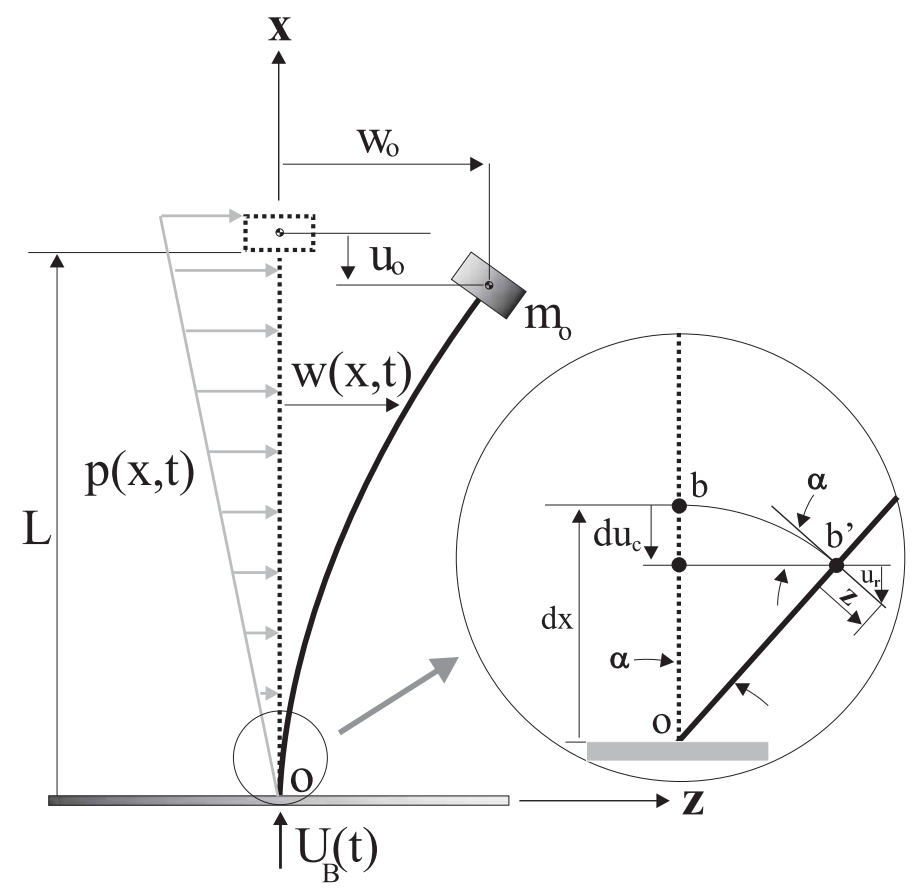

Fig. 2. Physical model of the structure under investigation, combined acoustic and base excitations.

\subsection{Kinematics of the beam-mass system}

Based on the structural system shown in Fig. 1, the physical model shown in Fig. 2 was considered. In this physical model, the $O X Y Z$ orthogonal coordinates system is fixed at the base of the beam in its unstressed position and, directed such as the $X$ axis is taken as the centreline of the beam. The origin $O$ of the coordinated system may be subject to a dynamic displacement, but only in the $X$ direction, described by $U_{B}(t)$. In addition, a distributed acoustic pressure is acting transversely in the $Z$ direction. A generalized coordinated $w(x, t)$ is used to measure the deformation from the unstressed position of an arbitrary axial position $x$ in the $Z$ direction.

When the structure is subject to transverse vibrations with finite amplitude, an imaginary transverse plane normal to the centerline of the beam at point $b$ translates in the $Z$ direction by an amount of $w(x, t)$, also translates in the 


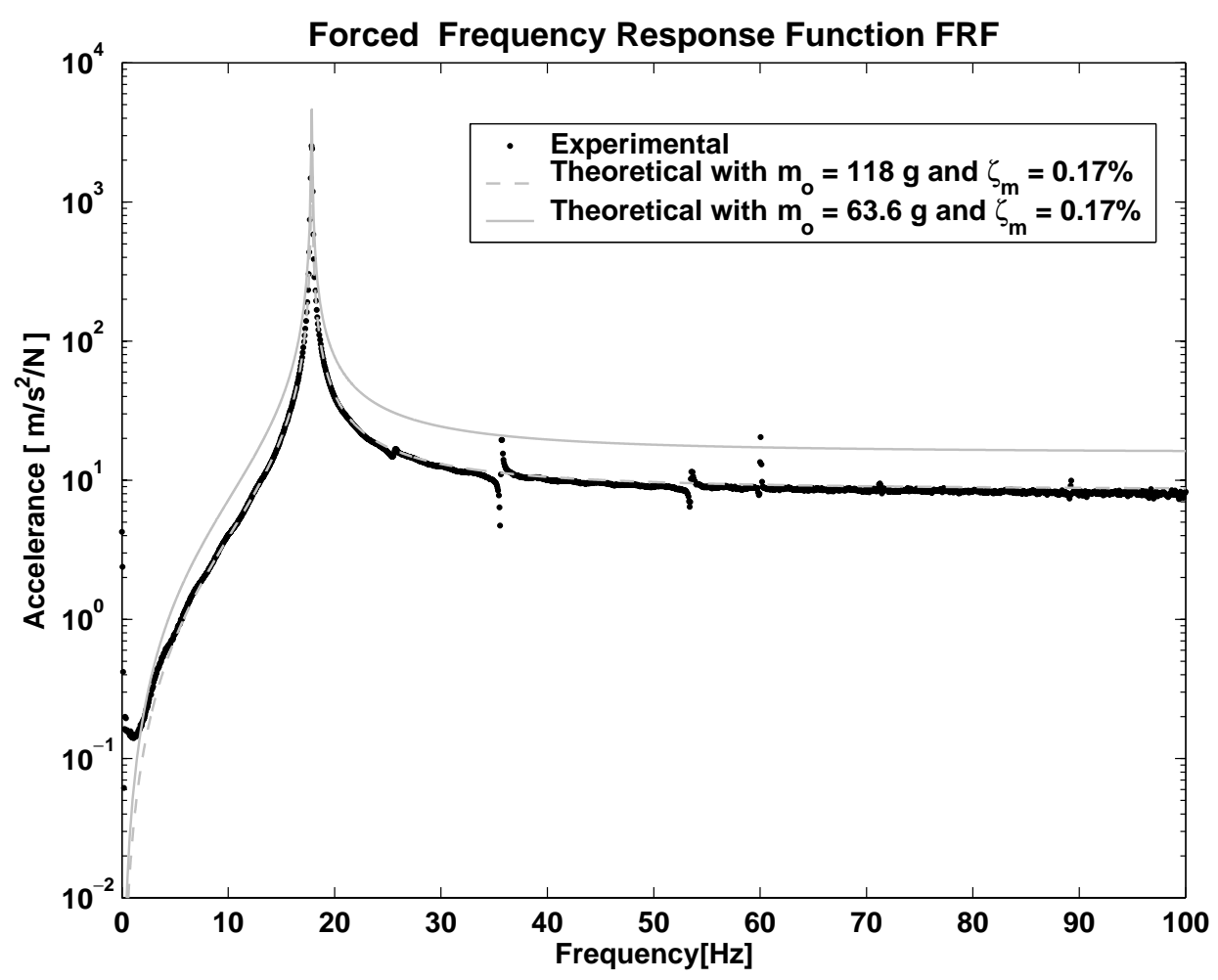

Fig. 3. Comparative results for the accelerance FRF for the test structure.

$X$ direction by an amount of $u_{c}$, and rotates in the $x z$ plane an amount given by the slope $\partial w / \partial x$ of the deflection curve at point $b$.

From Fig. 2 the tangent at the point $P^{\prime}$ is given by $\tan \alpha=\partial w / \partial x$. Then the angle $\alpha$ at the same point can be written in a series form as

$$
\alpha=\frac{\partial w}{\partial x}-\frac{1}{3}\left(\frac{\partial w}{\partial x}\right)^{3}+\frac{1}{5}\left(\frac{\partial w}{\partial x}\right)^{5}+\ldots
$$

In addition, the infinitesimal axial contraction $d u_{c}$ measured from the point $b$ at coordinate $(x, z)=(d x, 0)$ to the projection of the point $b^{\prime}$ into the $X$ axis is given as

$$
d u_{c}=d x(1-\cos \alpha)=d x\left(1-1+\frac{1}{2} \alpha^{2}-\frac{1}{24} \alpha^{4}+\ldots\right)
$$

From the modelling viewpoint it is interesting to express the axial contraction $d u_{c}$ as a function of the lateral displacement $w(x, t)$. Then substitution of Eq. (1) into Eq. (2) results in

$$
d u_{c}=\frac{1}{2} d x\left(\frac{\partial w}{\partial x}\right)^{2}-\frac{1}{3} d x\left(\frac{\partial w}{\partial x}\right)^{4}+\frac{1}{18} d x\left(\frac{\partial w}{\partial x}\right)^{6}+\ldots
$$

As mentioned earlier the rotation of any transverse section of the beam will produce an additional displacement in the $X$ direction. Accordingly, in considering the bending displacement in the $X$ direction of a point in a cross section of the beam at position $z$ below the centroidal axis in the undeformed geometry results in

$$
u_{r}=-z \sin \alpha=-z \alpha+\frac{1}{6} z \alpha^{3}-\frac{1}{120} z \alpha^{5}+\ldots
$$

or by employing Eq. (1), $u_{r}$ can be written as function of the lateral displacement $w(x, t)$ as 


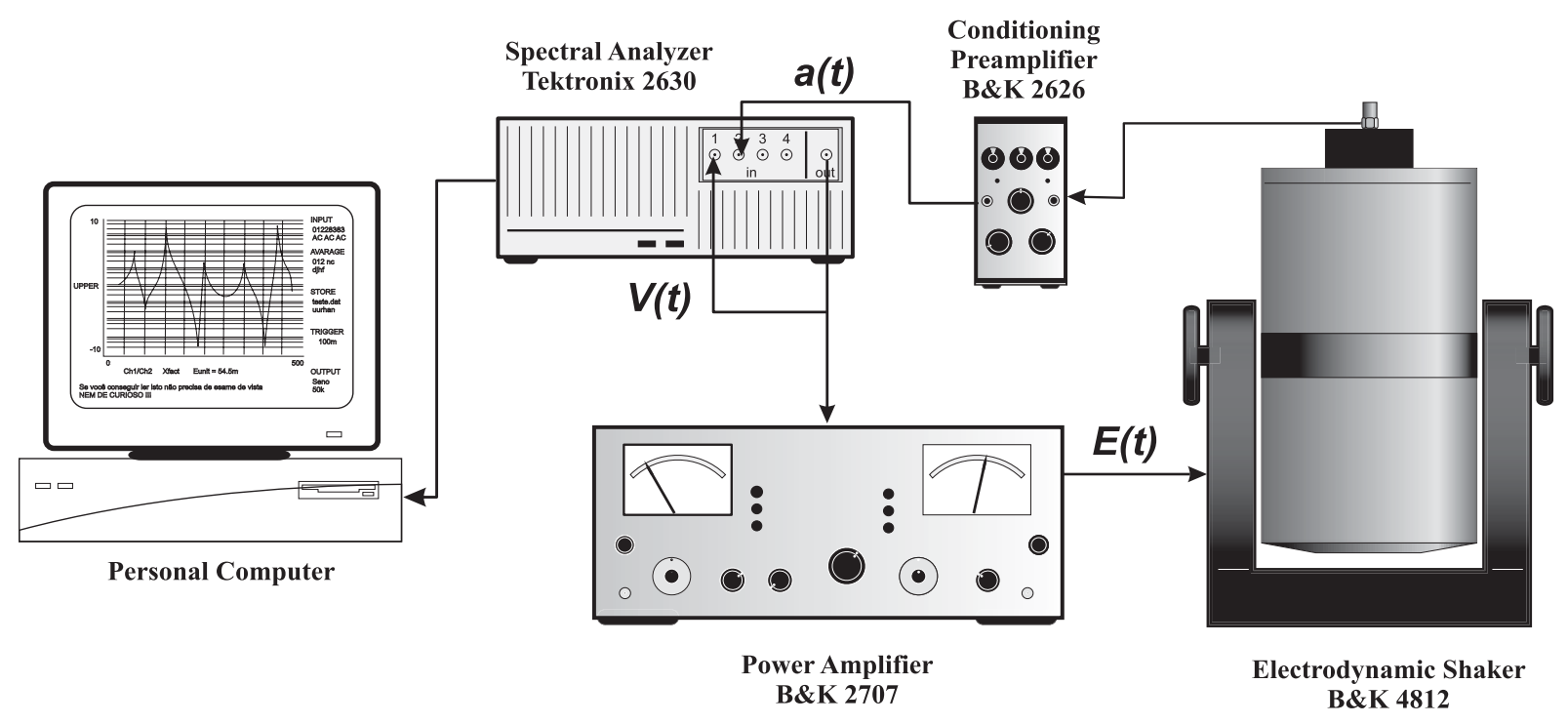

Fig. 4. Experimental setup arranged to perform the characterization of the base driven excitation source.

$$
u_{r}=-z \frac{\partial w}{\partial x}+\frac{1}{2} z\left(\frac{\partial w}{\partial x}\right)^{3}-\frac{7}{40} z\left(\frac{\partial w}{\partial x}\right)^{5}+\ldots
$$

The kinematic relationships just derived will be used next in obtaining the displacement field and later the strain and stress relationships.

\subsection{Displacement, strain and stress Fields}

The results just obtained from the kinematics along with the assumptions made in the beginning allow us to express the displacement field according to

$$
\left\{\begin{array}{l}
u_{1} \\
u_{2} \\
u_{3}
\end{array}\right\}=\left\{\begin{array}{c}
d u_{c}(x, t)+u_{r}(x, t)-U_{B}(t) \\
0 \\
w(x, t)
\end{array}\right\}
$$

which indicates that each transverse section of the beam has a displacement $u_{1}$ in the $X$ direction, does not present any displacement in the $Y$ direction, $u_{2}=0$, and has a displacement $u_{3}$ in the $Z$ direction. From the displacement field, the deformation field can be derived. In this derivation, the elasticity simplifying theory of small displacements [2] is used, then all the quadratic terms in the full strain equations are discarded. Following this theory, the strain components are written as

$$
\left[\begin{array}{lll}
\epsilon_{x x} & \gamma_{x y} & \gamma_{x z} \\
\gamma_{x y} & \epsilon_{y y} & \gamma_{y z} \\
\gamma_{x z} & \gamma_{y z} & \epsilon_{z z}
\end{array}\right] \cong\left[\begin{array}{ccc}
\frac{\partial u_{1}}{\partial x} & \frac{1}{2}\left(\frac{\partial u_{2}}{\partial x}+\frac{\partial u_{1}}{\partial y}\right) & \frac{1}{2}\left(\frac{\partial u_{3}}{\partial x}+\frac{\partial u_{1}}{\partial z}\right) \\
\frac{1}{2}\left(\frac{\partial u_{2}}{\partial x}+\frac{\partial u_{1}}{\partial y}\right) & \frac{\partial u_{2}}{\partial x} & \frac{1}{2}\left(\frac{\partial u_{3}}{\partial y}+\frac{\partial u_{2}}{\partial z}\right) \\
\frac{1}{2}\left(\frac{\partial u_{3}}{\partial x}+\frac{\partial u_{1}}{\partial z}\right) & \frac{1}{2}\left(\frac{\partial u_{3}}{\partial y}+\frac{\partial u_{2}}{\partial z}\right) & \frac{\partial u_{3}}{\partial x}
\end{array}\right]
$$

Therefore, by applying Eq. (7) into the displacement field equation and from $\mathrm{H}_{2}$ the strain field is obtained as

$$
\left[\begin{array}{ccc}
\epsilon_{x x} & \gamma_{x y} & \gamma_{x z} \\
\gamma_{x y} & \epsilon_{y y} & \gamma_{y z} \\
\gamma_{x z} & \gamma_{y z} & \epsilon_{z z}
\end{array}\right] \cong\left[\begin{array}{cccc}
\frac{\partial\left(d u_{c}\right)}{\partial x}+\frac{\partial u_{r}}{\partial x} & 0 & 0 \\
0 & 0 & 0 \\
0 & 0 & 0
\end{array}\right]
$$

Now, the stress field can be computed from Eq. (8). In the present case, it is considered that the beam is isotropic and homogeneous. Additionally it is also neglected the effects of the poisson ratio. Under these consideration, the 


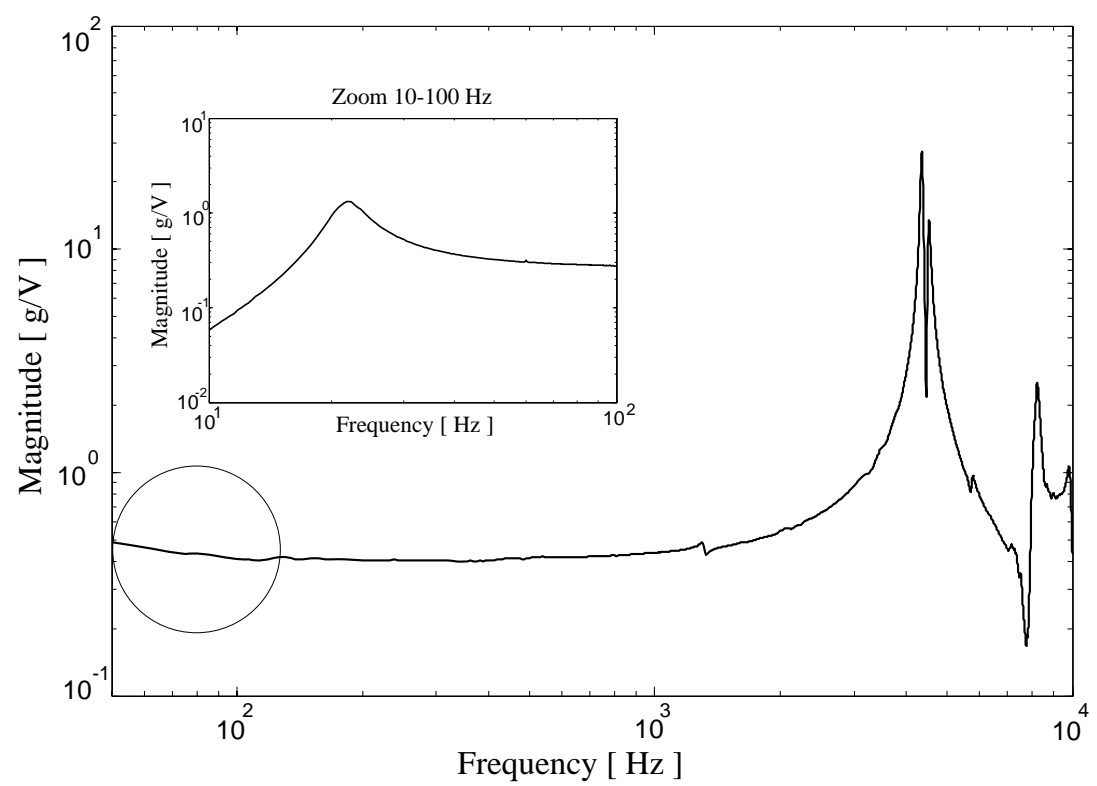

Fig. 5. Experimental shaker's frequency response function (FRF) showing amplifications and attenuations that can be reach in the response even using the same intensity as input.

stress field is simply given as

$$
\left[\begin{array}{lll}
\sigma_{x x} & \tau_{x y} & \tau_{x z} \\
\tau_{y x} & \sigma_{y y} & \tau_{y z} \\
\tau_{z x} & \tau_{z y} & \sigma_{z z}
\end{array}\right]=\left[\begin{array}{cccc}
E \frac{\partial\left(d u_{c}\right)}{\partial x}+E \frac{\partial u_{r}}{\partial x} & 0 & 0 \\
0 & 0 & 0 \\
0 & 0 & 0
\end{array}\right]
$$

in which $E$ is the Young modulus. Next, the energies of the system as well as the nonconservative generalized forces are obtained.

\subsection{Kinetic and strain energies and nonconservative generalized forces}

Considering the beam as a continuum solid with displacement field described by $u_{i}(i=1,2,3)$ and $u_{i}^{o}(i=1,2,3)$ the displacement when $x=L$, the complete kinetic energy of the lumped-mass system can be described by

$$
T=\frac{1}{2} \int_{V} \rho \dot{u}_{i} \dot{u}_{i} d V+\frac{1}{2} m_{o} \dot{u}_{i}^{o} \dot{u}_{i}^{o}
$$

in which the dot denotes time derivative, $\rho$ and $V$ are respectively the material density and volume of the beam, and $m_{o}$ is the value of the lumped mass. In order to simplify Eq. (10), the contribution of the distributed mass of the beam will be ignored $\left(H_{5}\right)$ as well as the rotatory energy of the lumped mass. Hence, the kinetic energy is simplified to

$$
T=\frac{1}{2} m_{o}\left[\left(\dot{u}_{1}^{o}\right)^{2}+\left(\dot{u}_{3}^{o}\right)^{2}\right]
$$

The task to find $T$ consists in performing several steps. First, the time derivative of the displacement field must be computed which results in expressions for $\left(\dot{u}_{1}\right)^{2}$ and $\left(\dot{u}_{3}\right)^{2}$. Second, the terms in the right hand side of the expressions of $\left(\dot{u}_{1}\right)^{2}$ and $\left(\dot{u}_{3}\right)^{2}$ are described as functions of the $w(x, t)$ and its spatial derivatives. Third, a spatial reduction is necessary so that the deflection on the center of the lumped mass can be obtained. This can be done by using a expression of form

$$
w(x, t)=\phi(x) w_{o}(t)
$$




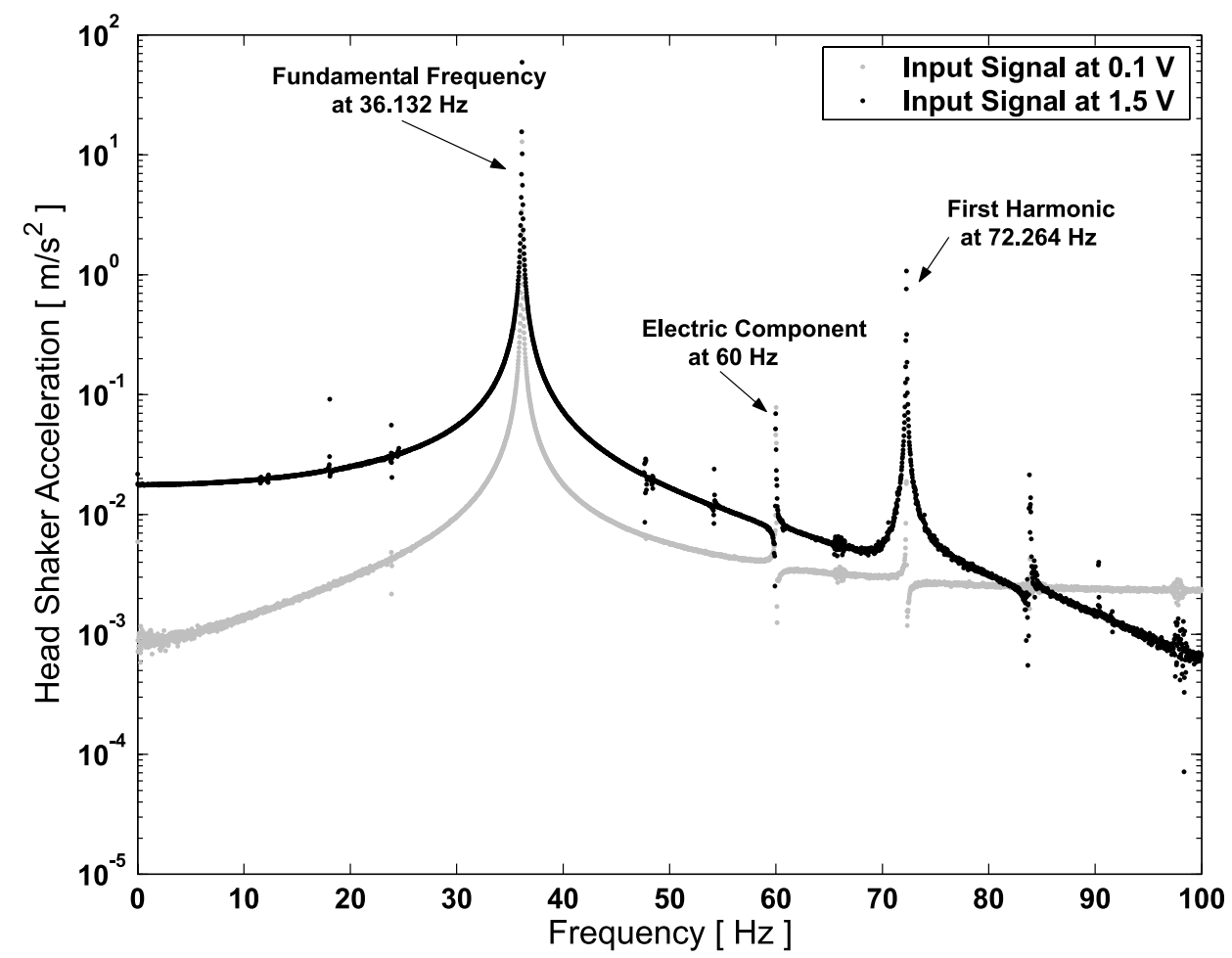

Fig. 6. Experimental result showing the presence of a harmonic in the shaker's response, even when the input (voltage) has got only one spectral component fixed at $36.132 \mathrm{~Hz}$.

in which $\phi(x)$ represents the first linear natural mode of the structure and $w_{o}(t)$ represents the modal coordinate associated with this natural mode. As a final result, the expressions for $\left(\dot{u}_{1}^{o}\right)^{2}$ and $\left(\dot{u}_{3}^{o}\right)^{2}$ are found and truncated to result in nonlinearities of third order

$$
\begin{aligned}
& \left(\dot{u}_{1}^{o}\right)^{2}=\left(A_{1}\right)^{2} w_{o}^{2} \dot{w}_{o}^{2}-2 A_{1} w_{o} \dot{w}_{o} \dot{U}_{B}+\frac{8}{3} A_{2} w_{o}^{3} \dot{w}_{o} \dot{U}_{B}+\left(\dot{U}_{B}\right)^{2} \\
& \left(\dot{u}_{3}^{o}\right)^{2}=\dot{w}_{o}^{2}
\end{aligned}
$$

Substitution of the expressions of $\left(\dot{u}_{1}^{o}\right)^{2}$ and $\left(\dot{u}_{3}^{o}\right)^{2}$ described above into Eq. (11), the kinetic energy is then given as

$$
T=\frac{1}{2} m_{o} \dot{w}_{o}^{2}+\frac{1}{2} m_{o}\left[\left(A_{1}\right)^{2} w_{o}^{2} \dot{w}_{o}^{2}-2 A_{1} w_{o} \dot{w}_{o} \dot{U}_{B}+\frac{8}{3} A_{2} w_{o}^{3} \dot{w}_{o} \dot{U}_{B}+\left(\dot{U}_{B}\right)^{2}\right]
$$

in which $A_{1}$ and $A_{2}$ are geometrical constants given as

$$
A_{1}=\int_{0}^{L}\left(\frac{\partial \phi}{\partial x}\right)^{2} d x
$$

and

$$
A_{2}=\int_{0}^{L}\left(\frac{\partial \phi}{\partial x}\right)^{4} d x
$$

and the first linear mode shape function $\phi(x)$ is given as

$$
\phi(x)=1-\cos \left(\frac{\pi x}{2 L}\right)
$$




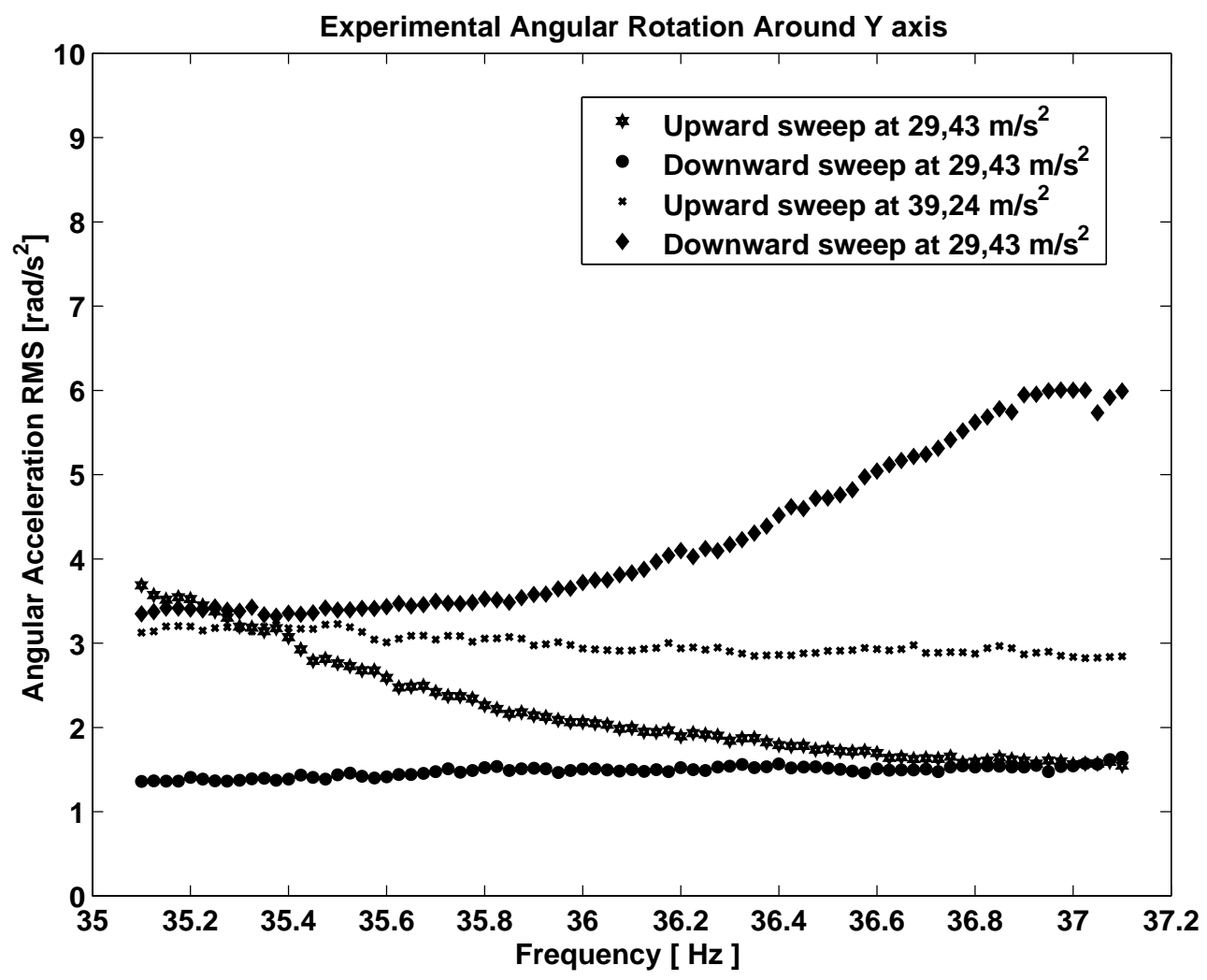

Fig. 7. Experimental result showing angular acceleration exhibited by the exciter armature.

Once the final expression for the kinetic energy is known, the next step towards the derivation of equation of motion is obtaining the system's strain or potential energy which may be written as function of the stress and strain as

$$
U=\frac{1}{2} \iiint_{V} \sigma_{i j} \varepsilon_{i j} d V \quad i, j=x, y, z
$$

From Eqs (8) and (9) the strain energy Eq. (19) for the system under consideration is reduced to

$$
U=\frac{1}{2} \iiint_{V} \sigma_{x x} \varepsilon_{x x} d V
$$

Still, using again the assumption that the material follow the constitutive Hooke's law, and ignoring the Poisson's effects, Eq. (19) reduces to

$$
U=\frac{1}{2} \iiint_{V} E \varepsilon_{x x}^{2} d V=\frac{1}{2} \int_{0}^{L} \int_{A} E \varepsilon_{x x}^{2} d A d x
$$

In the system's strain field, Eq. (21) the component $\varepsilon_{x x}$ is composed by the contribution of the total contraction displacement $u_{c}$ and $u_{r}$. However, $u_{c}$ is very small when compared to $u_{r}$. Therefore, to simplify the analysis, the contribution of $u_{c}$ and of the gravitational field to the strain energy are ignored, thus giving

$$
U=\frac{1}{2} \int_{0}^{L} \int_{A} E\left[z^{2}\left(w^{\prime \prime}\right)^{2}-z^{2}\left(w^{\prime \prime}\right)^{2}\left(w^{\prime}\right)^{2}\right] d A d x
$$

By writing $w(x, t)$ as a function of $\phi(x)$ and $w_{o}(t)$ (Eq. (12)), the final expression for the strain energy truncated cubic terms in the system's equation of motion is given by 


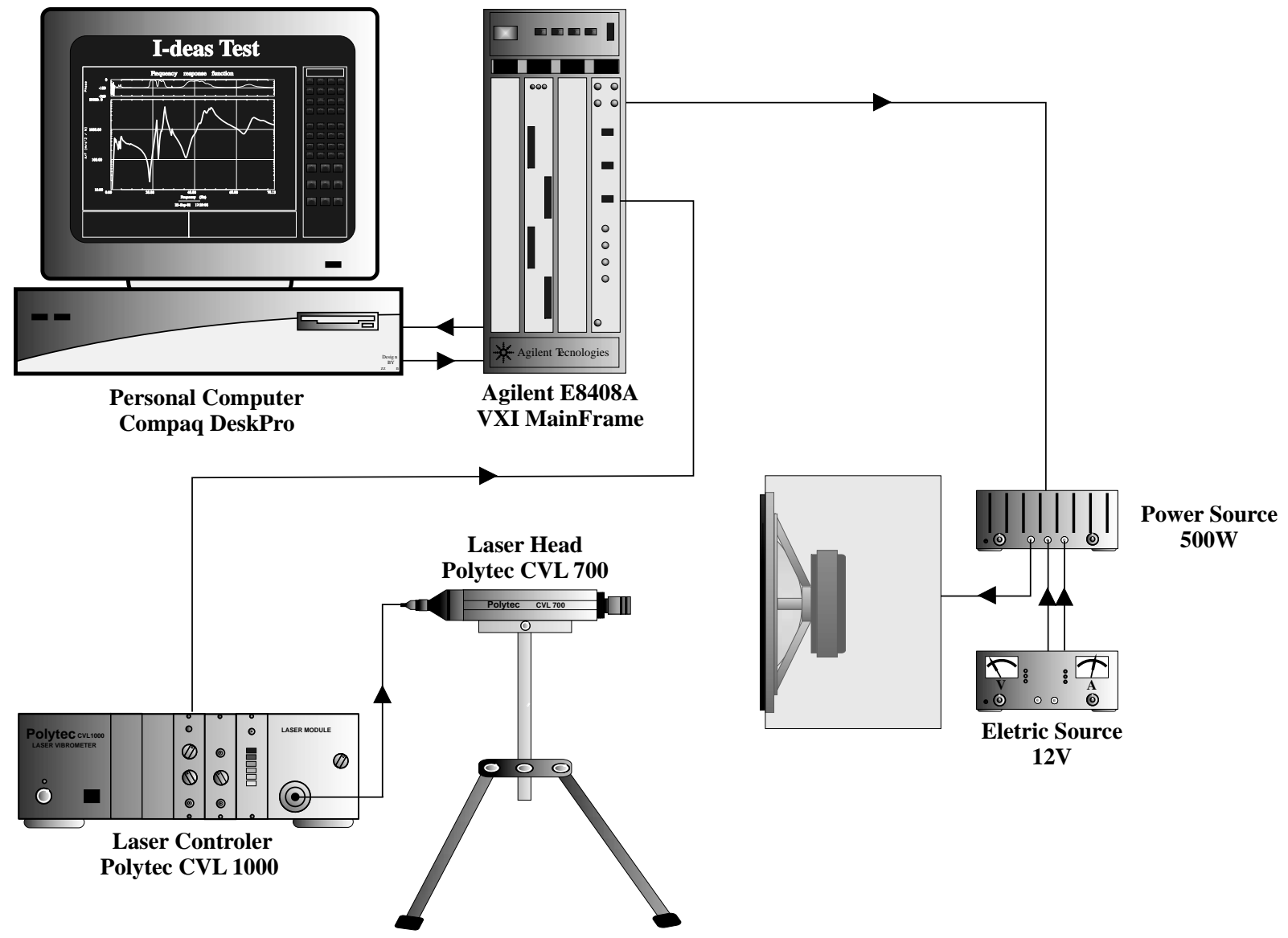

Fig. 8. Experimental setup for speed measurements of the speaker's cone.

$$
U=\frac{1}{2} E I_{y} B_{1} w_{o}^{2}-\frac{3}{2} E I_{y} B_{2} w_{o}^{4}+\ldots
$$

where $I_{y}$ is the area moment of inertial about the y axis and, the geometrical constants $B_{1}$ and $B_{2}$ are given as

$$
\begin{aligned}
& B_{1}=\int_{0}^{L}\left(\phi^{\prime \prime}\right)^{2} d x \\
& B_{2}=\int_{0}^{L}\left(\phi^{\prime \prime}\right)^{2}\left(\phi^{\prime}\right)^{2} d x
\end{aligned}
$$

The last step before deriving the system's equation of motion consists in obtaining the expression for the nonconservative forces acting on the system. Herein, it will be considered the action of three nonconservative forces. The first is the structural damping force which is modelled in terms of the generalized coordinates as $c_{1} \dot{w}_{o}$. The second is the aerodynamic drag damping force acting on the system (when in motion) and is proportional to the squared of the generalized velocity $c_{2} \dot{w}_{o}\left|\dot{w}_{o}\right|$. Both damping forces act in the negative direction of the virtual transversal displacement $\delta w_{o}$. The last nonconservative force is the acoustic excitation that is characterized as a single component sinusoidal force $F(t)$ applied at the center of gravity of the lumped mass and oriented along the $z$ direction. The external excitation $F(t)$ acts in the positive direction of the virtual transversal displacement $\delta w_{o}$. Physically $F(t)$ is originated from the dynamic pressure distribution over the structure $p(x, t)$, therefore $F(t)$ may be written as

$$
F(t)=\int_{A} p(x, t) \phi(x) d x
$$


Experimental Results at $36 \mathrm{~Hz}$

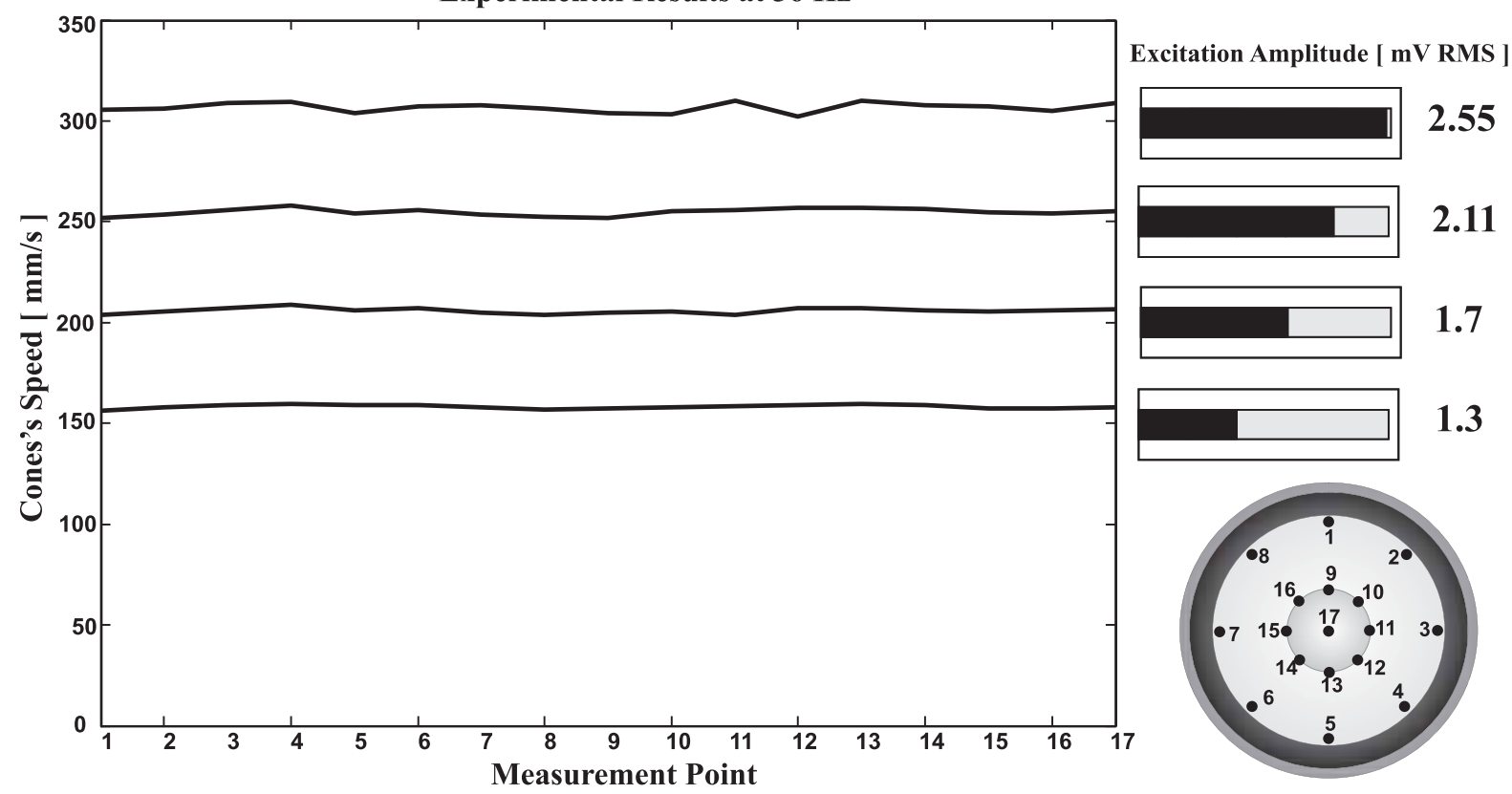

Fig. 9. Experimental speed measurements of the speaker's cone at $36.132 \mathrm{~Hz}$.

Therefore, the nonconservative virtual work $\delta W_{n c}$ which is done on the system is given by

$$
\delta W_{n c}=\left[-c_{1} \dot{w}_{o}-c_{2} \dot{w}_{o}\left|\dot{w}_{o}\right|+F(t)\right] \delta w_{o}
$$

Since the nonconservative virtual work is defined as a function of the nonconservative generalized force $Q_{n c}$ as $\delta W_{n c}=Q_{n c} \delta w_{o}$, the generalized force $Q_{n c}$ is obtained as

$$
Q_{n c}=\frac{\delta W_{n c}}{\delta w_{o}}=-c_{1} \dot{w}_{o}-c_{2} \dot{w}_{o}\left|\dot{w}_{o}\right|+F(t)
$$

\subsection{Equation of motion}

In the earlier section, the expressions for the kinetic energy $T$, strain energy $U$ and nonconservative generalized force $Q_{n c}$ were obtained. From these results, it possible to derive the system's equation of motion by using the well known Lagrange's equations [12], which in turn, for the system under investigation is written as

$$
\frac{d}{d t}\left(\frac{\partial T}{\partial \dot{w}_{o}}\right)-\frac{\partial T}{\partial w_{o}}+\frac{\partial U}{\partial w_{o}}=Q_{n c}
$$

Computation of each term of the Lagrange's equation and substitution of the result the Eq. (29) the following result is obtained

$$
\begin{aligned}
& \left(1+\underline{A_{1}^{2} w_{o}^{2}}\right) \ddot{w}_{o}+\left(\frac{c_{1}}{m_{o}}+\frac{c_{2}}{m_{o}}\left|\dot{w}_{o}\right|\right) \\
& w_{o}+\left(\underline{A_{1}^{2} \dot{w}_{o}^{2}}-\underline{A_{1} \ddot{U}_{B}}+\frac{E I_{y} B_{1}}{m o}\right) w_{o} \\
& +\left[\underline{\underline{\frac{4}{3} A_{2} \ddot{U}_{B}}}-\underline{\left.\underline{\left(\frac{6 E I_{y} B_{2}}{m o}\right)}\right] w_{o}^{3}=\frac{F(t)}{m o}}\right.
\end{aligned}
$$

Equation (30) represents an ordinary inhomogeneous nonlinear time-dependent differential equation. In addition, this equation holds both the axial contraction and the curvature nonlinear effects. If both the underlined and double underlined terms are ignored, this equation reduces to a classical linear damped forced model. On the other hand, 

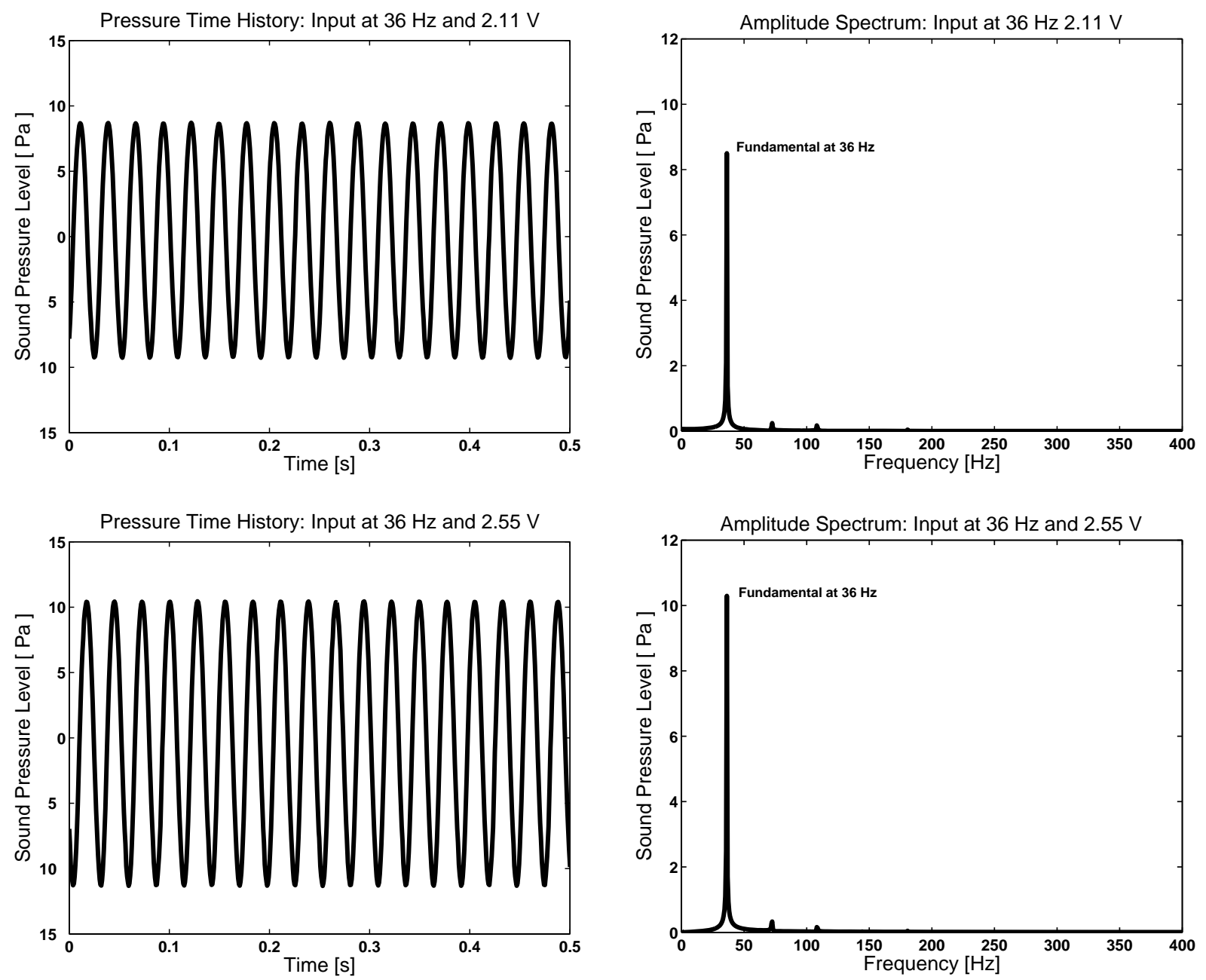

Fig. 10. Experimental measurements of the sound pressure level at input of $36 \mathrm{~Hz}$.

if only the double underlined terms are ignored this equation reduces to the same equation obtained in [9] plus a forced term. Still, if the double underlined terms plus the nonlinear damping were ignored, this equation reduces to the same model obtained by in [4] plus a forced term.

Since the present work is focused on the dynamic response of a structure under the effects of combined acoustic external and parametric excitation it is considered that these excitations are can be written as

$$
\ddot{U}_{B}(t)=-Q \lambda^{2} \cos (\lambda t+\varphi)=-Q_{o} \cos (\lambda t+\varphi)
$$

and

$$
F(t)=F_{o} \cos (\Omega t)
$$

in which $Q_{o}$ and $F_{o}$ are the magnitudes of the base acceleration and external force, respectively, $\lambda$ and $\Omega$ are the parametric and external excitation frequencies and $\varphi$ is the phase shift between the excitations. Then, Eq. (30) can be rewritten in the dimensional final form as

$$
\begin{gathered}
\left(1+A_{1}^{2} w_{o}^{2}\right) \ddot{w}_{o}+\left(\frac{c_{1}}{m_{o}}+\frac{c_{2}}{m_{o}}\left|\dot{w}_{o}\right|\right) \dot{w}_{o}+\left(A_{1}^{2} \dot{w}_{o}^{2}+A_{1} Q_{o} \cos (\lambda t+\varphi)+\frac{E I_{y} B_{1}}{m o}\right) \\
w_{o}+\left[-\frac{4}{3} A_{2} Q_{o} \cos (\lambda t+\varphi)-\frac{6 E I_{y} B_{2}}{m o}\right] w_{o}^{3}=\frac{F_{o}}{m o} \cos (\Omega t)
\end{gathered}
$$




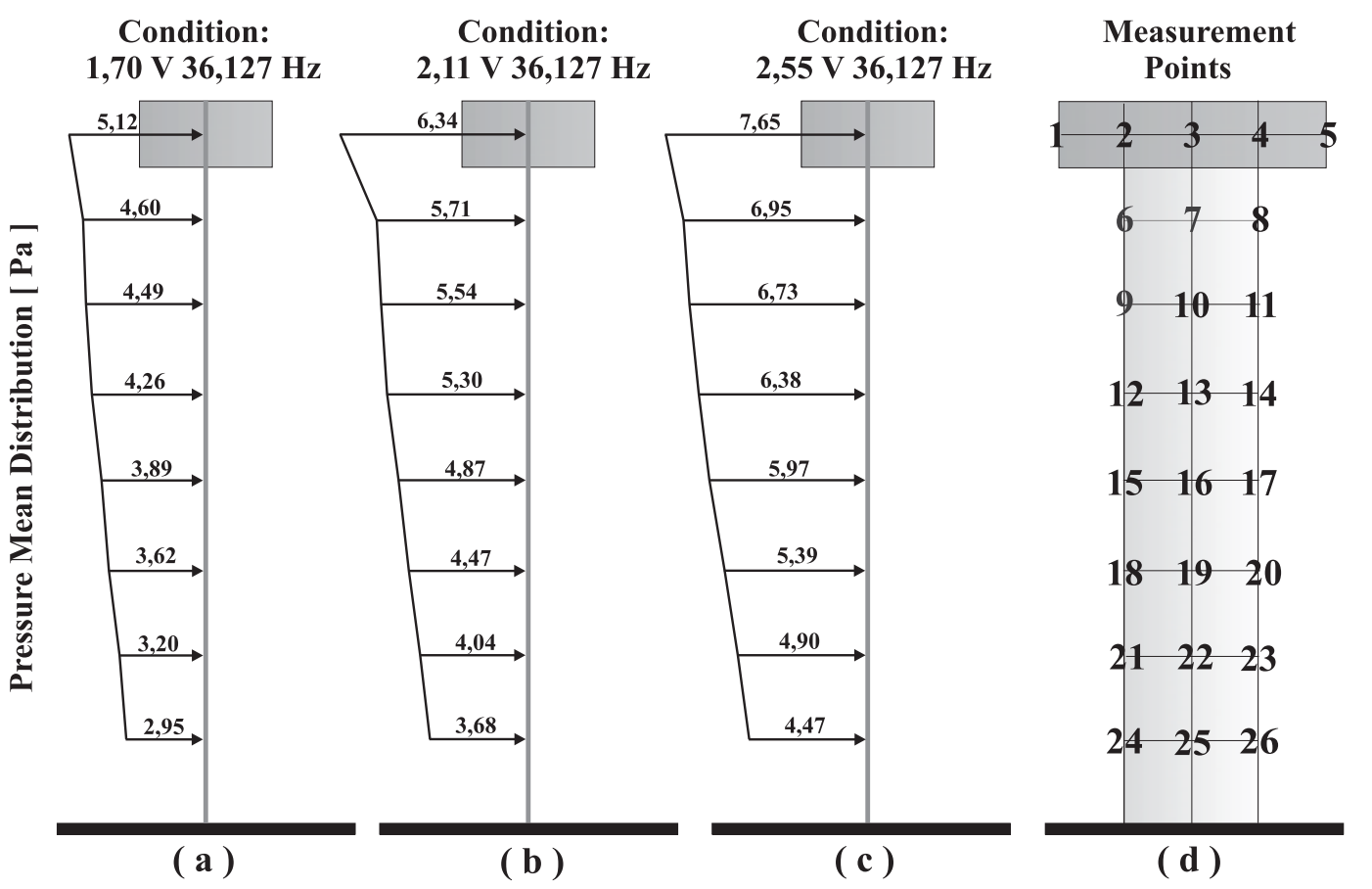

Fig. 11. Experimental pressure distribution over the structure for acoustic source's input at $36.127 \mathrm{~Hz}$ and three different amplitudes: (a) $1.70 \mathrm{~V}$; (b) $2.11 \mathrm{~V}$; (c) $2.11 \mathrm{~V}$ and (d) measured points.

From the numerical viewpoint it is interesting to work with the differential equation in dimensionless form. Therefore, by setting new dimensionless variables $w_{o}^{*}=A_{1} w_{o}$ and $t^{*}=t / T_{n}$ in which $T_{n}$ is the period of free vibration, the system's equation of motion is given by

$$
\begin{gathered}
\left(1+w_{o}^{* 2}\right) \ddot{w}_{o}^{*}+H_{1} \dot{w}_{o}^{*}+H_{2} \dot{w}_{o}^{*}\left|\dot{w}_{o}^{*}\right|+\left[1+\dot{w}_{o}^{* 2}+H_{3} \cos \left(\Theta t^{*}+\varphi\right)\right] w_{o}^{*}- \\
-\left[H_{5}+H_{4} \cos \left(\Theta t^{*}+\varphi\right)\right] w_{o}^{* 3}=H_{6} \cos \left(\Psi t^{*}\right)
\end{gathered}
$$

in which

$$
\begin{gathered}
H_{1}=\frac{c_{1} T_{n}}{m_{o}} ; \quad H_{2}=\frac{c_{2}}{m_{o} A_{1}} ; \quad H_{3}=Q_{o} A_{1} T_{n}^{2} \\
H_{4}=\frac{4 A_{2} Q_{o} T_{n}^{2}}{3 A_{1}^{2}} ; \quad H_{5}=\frac{6 B_{2} E I_{y} T_{n}^{2}}{m_{o} A_{1}^{2}} ; \quad H_{6}=\frac{F_{o} A_{1} T_{n}^{2}}{m_{o}} \\
\Theta=\lambda T_{n} ; \quad \Psi=\Omega T_{n}
\end{gathered}
$$

\section{Structure modal identification}

In a previous paper [9] a detailed modal testing is performed on the structure shown in Fig. 1 by employing a transient excitation signal to obtain the structure's frequency response function in the vicinity of the first beam's bending natural frequency.

Figure 3 exhibits predicted FRFs for two different values for the lumped-mass $m_{o}$. The first value used comes from the lumped-mass plus the clamp bolts giving an equivalent mass of $63.6 \mathrm{~g}$. The second value used $\mathrm{m}_{\mathrm{o}}=118 \mathrm{~g}$ comes from the best value of the lumped-mass in order to match with the experimental result, which can be claimed that for this value the theoretical and experimental curves matched very closely in the entire frequency range. On the other hand, the same behavior does not occur for the case where $m_{o}=63.4 \mathrm{~g}$, even though this result also presents good agreement with the measured results. The theoretical result depicted on Fig. 3 was obtained from basic theory and the system's modal parameters [9]. 
Experimental Response-Amplitude Curve

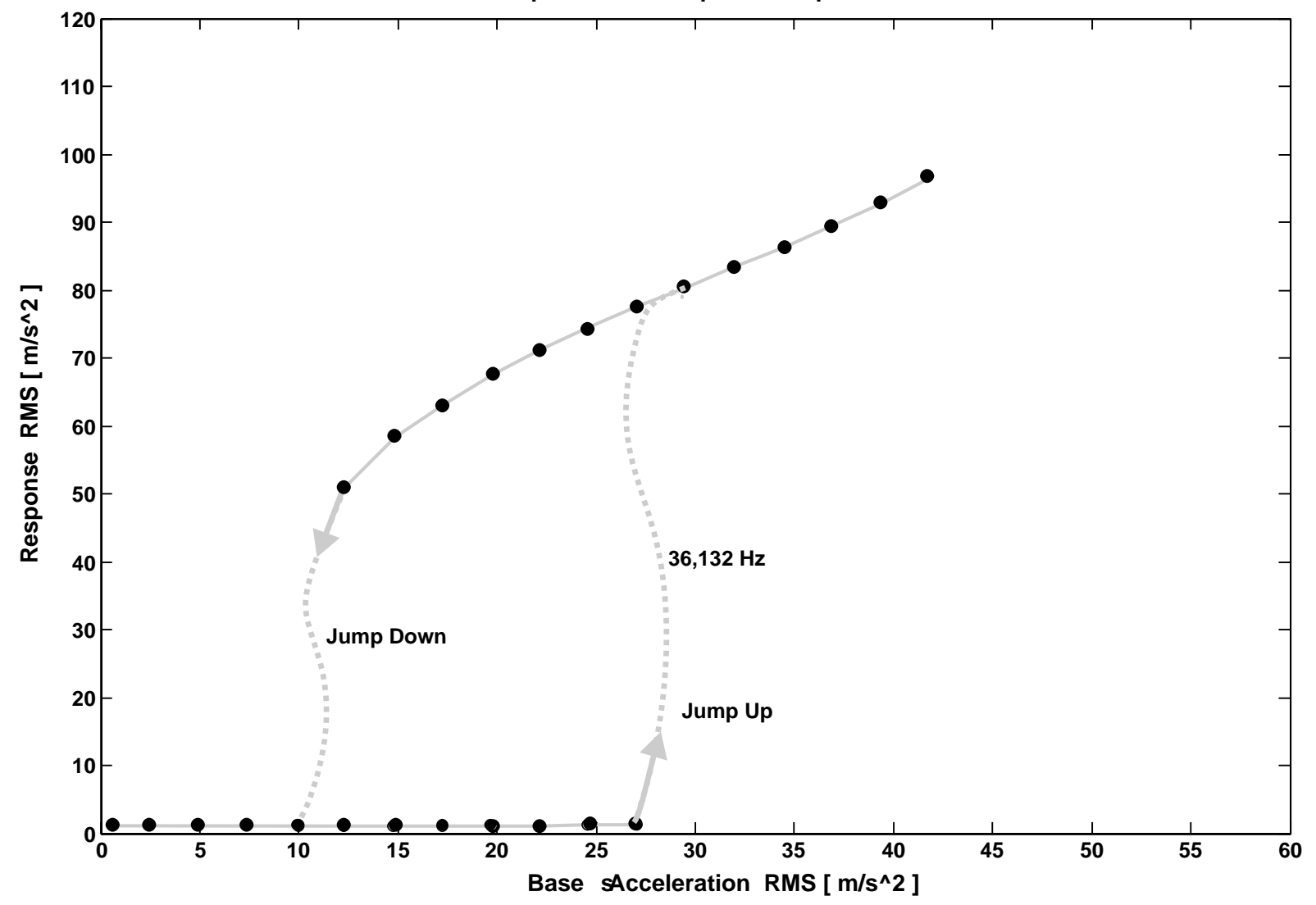

Fig. 12. Experimentally determined response-amplitude curve without the presence of external excitation for principal parametric resonance case.

\section{Experimental characterization of the excitation sources}

Most excitation mechanisms are essentially non ideal since they tend to present some interaction with the test structure [16,17]. In some cases, this interaction might alter significantly the test results. Therefore, detailed knowledge of the excitation sources is vital in conducting vibration tests, specially when nonlinear behavior is observed, as it is the present case. Hence, this section is devoted to discuss some important characteristics of the two excitation mechanisms used in the investigation, the parametric and acoustic excitations.

\subsection{The base driven excitation source}

The base driven excitation signal is provided by the B\&K 4812 general purpose vibration exciter. In order to assess not only the quality of the excitation signal but most importantly any considerable exciter-structure interaction during the tests, a series of preliminary tests are performed on the exciter without the presence of the test structure, according to the setup shown in Fig. 4, where the steel base mass used to attach the lumped-mass system is mounted to the exciter's table

The first test consisted of a sine sweep covering the $0-10 \mathrm{KHz}$ frequency range, and the exciter's table acceleration response frequency spectrum was measured by a miniature B\&K 4374 accelerometer and processed by the Tektronix 2630 spectrum analyzer. A total of 4096 data points were captured in each time period with a frequency resolution of $\triangle f=6.25 \mathrm{~Hz}$. The result of this test is shown in Fig. 5 where the amplitudes are scaled by the power amplifier input voltage in order to get an FRF in g's of acceleration of the base per unit of Volts. 

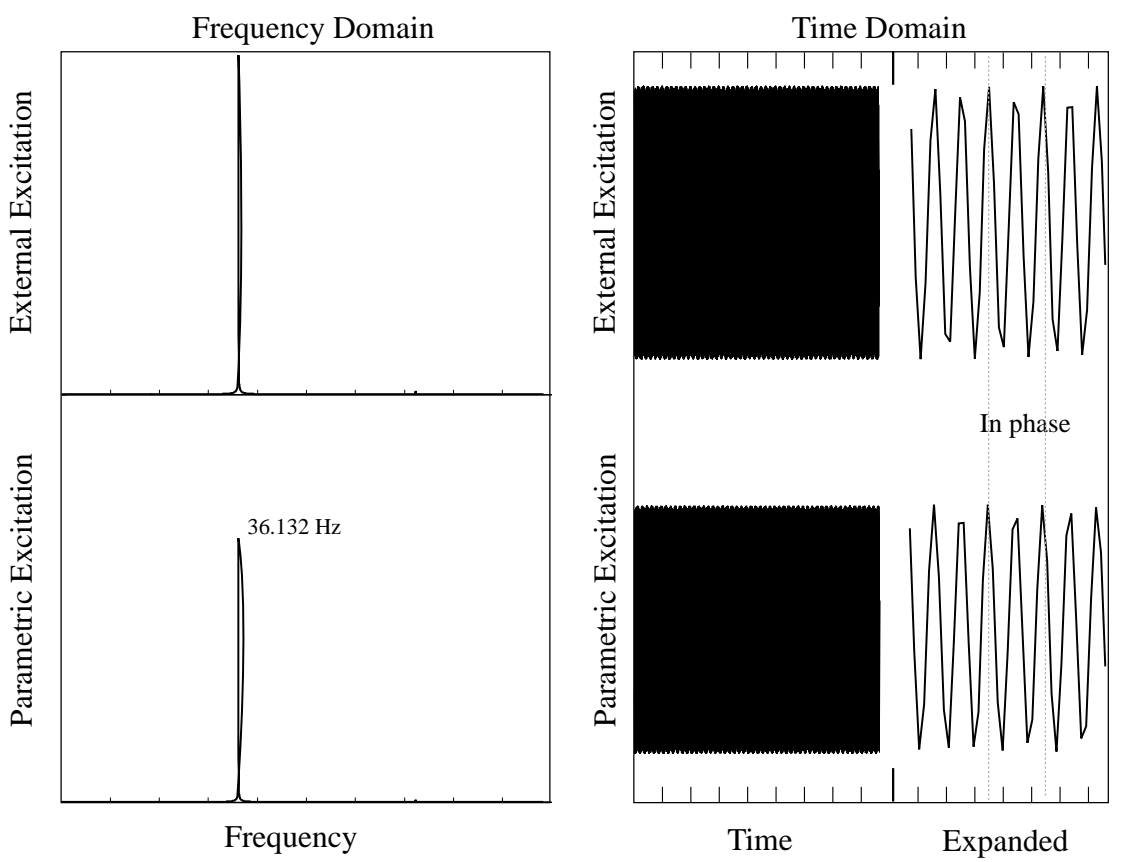

Fig. 13. Experimental frequency and time domains representation of the excitations, for the case of they are in phase shift.

From Fig. 5 it is first observed a decrease of the exciter bare table resonance from the nominal value of $7.2 \mathrm{KHz}$ to $4.2 \mathrm{KHz}$ due to the large mass attached to exciter table. This represents no problem to the present nonlinear study since here only the first $200 \mathrm{~Hz}$ are covered and the new $4.2 \mathrm{KHz}$ resonant frequency is well beyond this range. Figure 5 still shows a zoom in the $10-100 \mathrm{~Hz}$ which reveals the exciter suspension resonant frequency at approximately $22 \mathrm{~Hz}$. This resonance frequency alters the amplitude value of the exciter response FRF what can further affects the results of the forthcoming nonlinear tests. Therefore, a control strategy is need in the nonlinear tests such that the armature's response is maintained constant in the test frequency range.

Figure 6 shows the result for the second test performed with the exciter in the absence of the test structure. In this case the exciter was driven at a fixed single component sinusoidal frequency of $36.132 \mathrm{~Hz}$, which will be further used in the nonlinear tests. By keeping the power amplifier gain at a constant value the table's response was measured for two input voltages selected in the spectrum analyzer, which are $V(t)=0.1 \mathrm{~V}$ and $V(t)=1.5 \mathrm{~V}$ respectively. The results shown in Fig. 6 indicates a major response at $36.132 \mathrm{~Hz}$ in both cases, with amplitudes of $9.8 \mathrm{~m} / \mathrm{s}^{2}$ and $39 \mathrm{~m} / \mathrm{s}^{2}$, respectively. The results also exhibit a second harmonic at approximately $72.264 \mathrm{~Hz}$. The second harmonic presents different amplitude levels for the two input voltages being considerably larger for the higher input voltage value. The presence of higher harmonics was previously observed by Tomlinsom [16] in a theoretical and experimental investigation. The major conclusion of this work relates the presence of higher harmonics in the excitation signal due to the nonlinear nature of the exciter's magnetic field.

Figure 7 shows the results for the last test performed on the exciter's table. Four sweep runs were performed by using the sine-dwell and in a narrow frequency range in the vicinity of $36 \mathrm{~Hz}$ and with controlled amplitude. The first two runs were performed with the excitation amplitude fixed and controlled at $29.43 \mathrm{~m} / \mathrm{s}^{2}$. The first run was performed sweeping the excitation frequency upward while the second downward. The last two runs were performed similarly, but using a higher excitation amplitude value of $39.24 \mathrm{~m} / \mathrm{s}^{2}$. In all tests it was observed some angular motion of the exciter's table, however at modest values. The highest angular acceleration value was obtained in the downward sweep direction for the excitation amplitude of $39.24 \mathrm{~m} / \mathrm{s}^{2}$. On the other hand, the lowest value for the angular acceleration was observed also in the downward sweep direction but for the excitation amplitude of $39.24 \mathrm{~m} / \mathrm{s}^{2}$. 

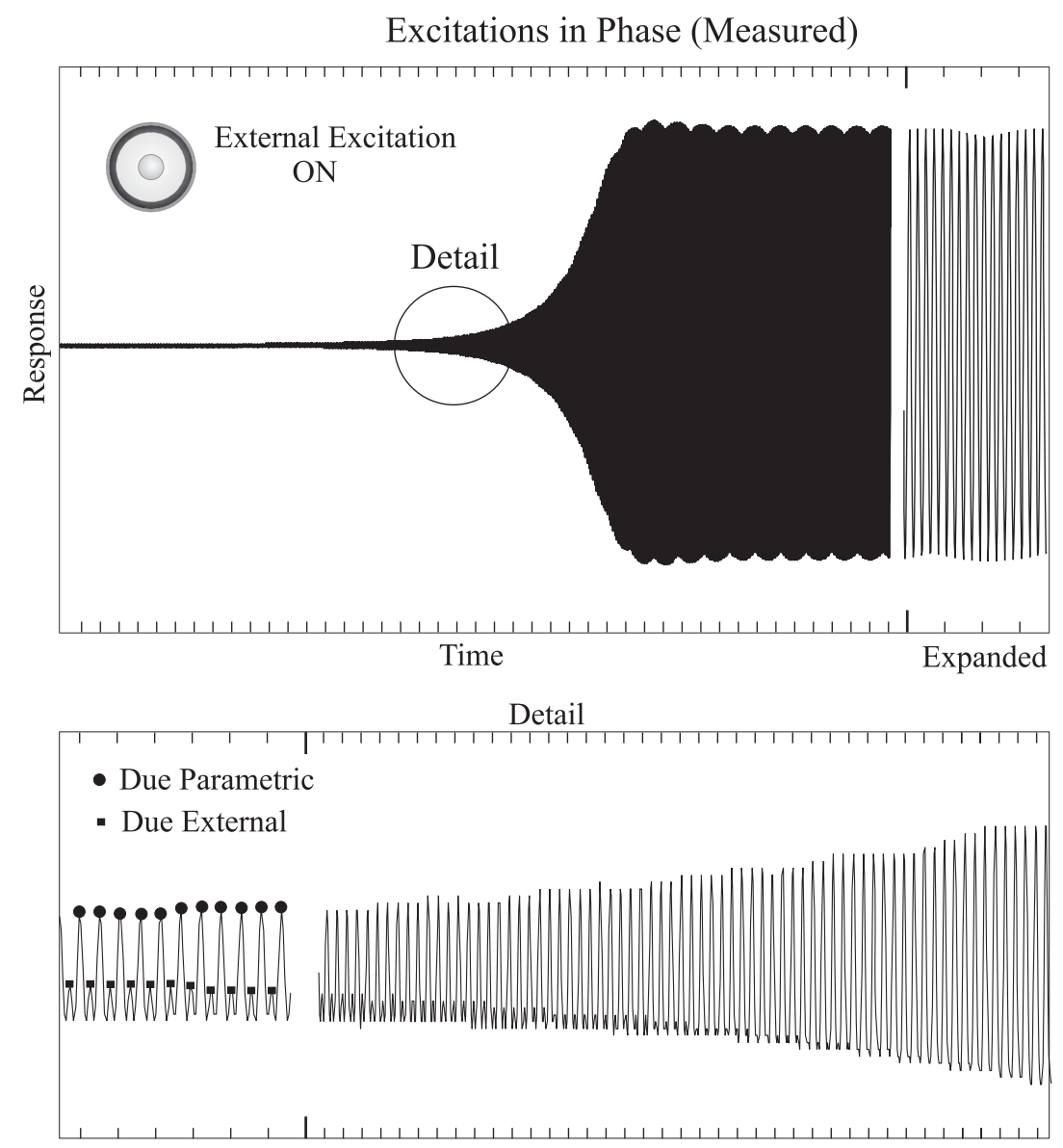

Fig. 14. Measured time-history, with the effects of combined parametric and external excitations, both in phase.

\subsection{The acoustic excitation source}

The acoustic excitation system used in the laboratory tests consists of a sealed 12" loudspeaker driven by a commercially available $500 \mathrm{~W}$ power amplifier. As previously discussed for the base driven source, it is of fundamental importance to conduct some preliminary tests on the acoustic source for two major reasons: (i) Since the input sound pressure is a distributed load in nature, it can be significantly altered by the structure's geometry; (ii) The excitation signal provided by the speaker when driven by a sinusoidal signal at a fixed frequency usually tends to present higher harmonics. Hence, two tests were performed, the first to check the excitation levels and quality of the acoustic input signal, and the second to check the presence of significant higher harmonics in the acoustic excitation signal, what could violates the assumption that the structure is being driven by a single frequency sinusoidal signal.

Figure 8 depicts the experimental arrangement that was used in the first test. Here, the enclosed speaker is suspended by flexible cords in order to suppress possible interference from the neighborhood. The speaker is then driven by a fixed sinusoidal signal and the speaker's membrane velocity is measured by a Polytec CLV 1000 $(50.0(\mathrm{~mm} / \mathrm{s}) / \mathrm{V})$ laser vibrometer at seventeen points distributed on the speaker's membrane surface, as indicated in Fig. 8. The test is then repeated for different input voltages at a frequency of approximately $36 \mathrm{~Hz}$. The measured velocity signals were sent to the Tektronix 2630 analyzer and the RMS values were calculated.

Figure 9 and Table 1 show the experimental results obtained from this test. It can be clearly seen from this results that the speaker presents a uniform cone velocity for the different input voltage levels as shown in Fig. 9. Table 1 shows some numerical results obtained from the signals showed in Fig. 9, where it can be noticed a small value for the standard deviation of each individual measurement, what reinforces the assumption of rigid behavior for 


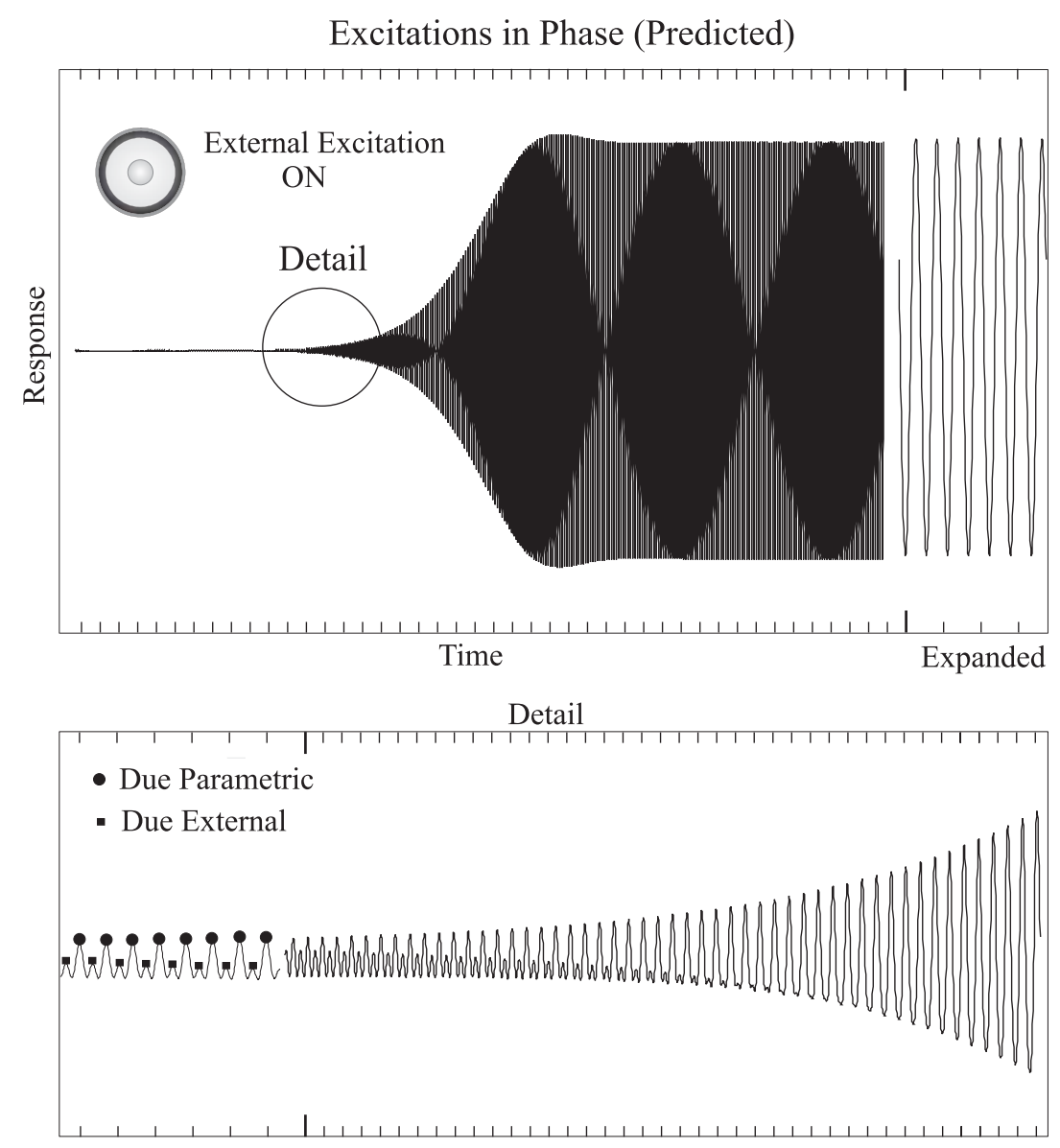

Fig. 15. Predicted time-history, with the effects of combined parametric and external excitations, both in phase.

the speaker's cone.This is a highly desirable result since it is a reasonable indication that the speaker cone can be considered as rigid surface forcing the fluid particles to move towards the structure, as indicated in [7].

In the second test, an experimental setup identical to the one previously used and shown in Fig. 8 was employed, except that in this case the laser vibrometer was replaced by a 1/2" G.R.A.S. 40AE microphone (49.49 mV/Pa) positioned at about $150 \mathrm{~mm}$ from the center of the loudspeaker. Despite the large volume available in the test room, foam wedges were positioned in the vicinity of the test setup in order to reduce sound wave reflections that could affect the test results. The main goal of this test is to check possible distortions in the acoustic signal and possibly the presence of higher harmonics. Two sinusoidal excitation frequencies were chosen, $18 \mathrm{~Hz}$ and $36 \mathrm{~Hz}$, respectively. The first frequency is very close to the first bending natural frequency of the test structure while the second is located in the vicinity of the base driven parametric excitation frequency. Figure 10 depicts the test results for the excitation frequency at $36 \mathrm{~Hz}$ and two input voltage values.

The results indicate that an excellent acoustic excitation signal is obtained when the speaker is driven at the parametric excitation frequency, since the time domain signal presents nearly no distortion due to higher harmonics. Although not shown here, the same behavior was not noticed when the speaker was driven at $18 \mathrm{~Hz}$ since the resulting measured sound pressure signal presented significant distortion mostly due to the appearance of the first harmonic at $36 \mathrm{~Hz}$. Therefore, the idea of simultaneously applying the base driven and acoustic sinusoidal signals at respectively $36 \mathrm{~Hz}$ and $18 \mathrm{~Hz}$ was definitively abandoned and both excitation sources were operated at a same frequency very close to the parametric frequency, $36 \mathrm{~Hz}$ by two independent signal generators.

The last result shown in this section is indicated in Fig. 11 and corresponds to the sound pressure distribution over the structure for different input voltages. In this case, the loudspeaker is driven by a sinusoidal signal at $36 \mathrm{~Hz}$ and 
the sound pressure is measured by a $1 / 2$ " microphone in the vicinity of the beam. It can be claimed that for all three levels of inputs the shape of the sound pressure is nearly the same.

Since a pressure distribution is needed in Eq. (26) the results shown in Fig. 11 were curve fitted using a linear function and the following results were obtained and used with Eq. (26)

$$
\begin{aligned}
& p_{170}(x)=22.6 x+2.67 \\
& p_{211}(x)=27.4 x+3.37 \\
& p_{255}(x)=33.0 x+4.08
\end{aligned}
$$

\section{Nonlinear tests results and comparison with theory}

This section shows the results obtained from the nonlinear tests and a comparative analysis with numerically simulated results that were generated from the theoretical model in the previous sections. A total of three nonlinear tests were planed and conducted, as described below:

NLT1 Exciting the structure using only the parametric excitation at $\lambda=2 \omega_{n}$ (principal parametric resonance case) for several amplitude levels $Q_{o}$.

NLT2 Exciting the structure using both the external and parametric excitations, fixing $\lambda=\Omega=2 \omega_{n}$, and for several amplitude levels of the parametric excitation $Q_{o}$, and for three amplitude levels of the external excitation $F_{o}$, keeping the excitation signals in phase.

NLT3 It is a test similar to the NLT2, however fixing the excitation signals out of phase.

Figure 12 shows the classical amplitude-response curve obtained from the experiments by following the strategy of test NLT1. This test was preformed by increasing the amplitude of parametric excitation from 0 to $44.5 \mathrm{~m} / \mathrm{s}^{2}$ $R M S$ while the frequency $\lambda$ was kept fixed at $36.132 \mathrm{~Hz}$ and then lowering the excitation amplitude at the same rate. A close look at this result reveals that between 25 and $30 \mathrm{~m} / \mathrm{s}^{2} R M S$ the structure exhibits the principal parametric resonance phenomenon, and consequently a very high response amplitude is developed about $75 \mathrm{~m} / \mathrm{s}^{2} R M S$. In addition, this figure still shows that the structure responds differently depending on the sweeping direction of the excitation amplitude. The result shown in Fig. 12 along with other experimental results obtained in the absence of the external acoustic excitation and not shown here were important to calibrate the analytical model previously obtained for the test structure and that will be used here along with the experimental results for comparison purposes.

\subsection{Case 1: $\Omega=\lambda=2 \omega_{n}$}

In this subsection, the calibrated mathematical model will be compared with the measured data for two different situations, when the excitations (parametric and external) are in phase; and when the excitations are out of phase. In both cases, $\Omega=\lambda=2 \omega_{n}$. Before the presentation of the results, it is required to check whether the excitation signals are maintained in phase or not by the instrumentation.

Figure 13 shows the experimentally obtained frequency and time domain representations of the excitations when the signals are adjusted in phase in the instrumentation. From this figure, it can be seen that the structure was indeed excited under this condition, which means that no significant distortion is introduced by the instrumentation that could possibly corrupt the required zero phase requirement between the excitation signals.

Figures 14 and 15 show the experimental and numerically simulated results corresponding to the situations where the excitation signals are maintained in phase (NLT2).

A close examination in the detailed portion of the response reveals the interaction between the parametric and external excitation sources. With the development of the principal parametric resonance, the structure responds at its first bending natural frequency frequency $\omega_{n}=18 \mathrm{~Hz}$, plus harmonics in a increasing amplitude. Simultaneously, due to the presence of a external excitation the structure also responds, however at a frequency equal to external excitation $\Omega=36 \mathrm{~Hz}$ plus harmonics. When the ratio between the external response to parametric response is high there is a clear separation of these components, on the other hand, when this ratio is small, there is a merge of these response contributions and since the parametric resonance causes significantly higher vibration amplitudes, it prevails once the steady state regime is reached. A qualitative comparison between the experimental and numerically simulated results from Figs 14 and 15 shows a reasonably good agreement. Very similar numerical and experimental results were obtained when the excitation signals are out of phase (NLT3). 
Table 1

Speaker Cone's speed using excitation frequency for various amplitude levels

\begin{tabular}{ccccc}
\hline Input Level $[\mathrm{mV}]$ & Max $[\mathrm{mm} / \mathrm{s}]$ & Min $[\mathrm{mm} / \mathrm{s}]$ & Mean $[\mathrm{mm} / \mathrm{s}]$ & STD $[\mathrm{mm} / \mathrm{s}]$ \\
\hline 1.3 & 159.6 & 156.4 & 158.2 & 1.00 \\
1.7 & 208.7 & 203.7 & 205.9 & 1.34 \\
2.11 & 258.1 & 252.0 & 254.8 & 1.80 \\
2.55 & 310.0 & 302.0 & 306.6 & 2.46 \\
\hline
\end{tabular}

Table 2

Dimensionless parameters used in the numerical simulations

\begin{tabular}{cccccc}
\hline $\mathrm{H}_{1}$ & $\mathrm{H}_{2}$ & $\mathrm{H}_{3}$ & $\mathrm{H}_{4}$ & $\mathrm{H}_{5}$ & $\mathrm{H}_{6}$ \\
\hline 0.0077 & 0.1547 & 1.7132 & 2.7820 & 51.1779 & $0.47 ; 1.43 ; 1.90$ \\
\hline
\end{tabular}

\subsection{Case 2: $\Omega$ and $\omega_{n}$ commensurable and $\lambda=2 \omega_{n}$}

In this item, the interaction between the external and parametric excitations as well as their consequences on the structure dynamics for the general case when $\Omega$ and $\omega_{n}$ are commensurable and $\lambda=2 \omega_{n}$ are shown and discussed. In order to simplify the analysis, the system's natural frequency $\omega_{n}=18.066 \mathrm{~Hz}$ is approximated to $\bar{\omega}_{n}=20 \mathrm{~Hz}$ in order to conduct the numerical simulations. In this case the external excitation $\Omega$ and $\bar{\omega}_{n}$ are commensurate, that is, there exist non-zero integers (positives or negatives) $k_{i}$ such that $k_{1} \Omega+k_{2} \bar{\omega}_{n}=0$, or in terms of the the parametric excitation $\lambda$ it is equal to $k_{1} \Omega+1 / 2 k_{2} \lambda=0$ (for the principal parametric case).

After the above preliminary observations two simulations were performed using the developed mathematical model. In the first simulation, it was considered the ratio $\Omega: \bar{\omega}_{n}=1: 20$ and for three external excitation amplitudes, represented by the dimensionless coefficient $H_{6}$. The second simulation was performed for $\Omega: \bar{\omega}_{n}=3: 20$, and for the same three dimensionless coefficients $H_{6}$. Table 2 shows the dimensionless parameters used in the simulation.

Figure 16 shows the resulting predictions when $\Omega: \bar{\omega}_{n}=1: 20$ and for three values of $H_{6}=0.47, H_{6}=1.43$ and $H_{6}=1.90$. The central portion of this figure shows the complete time-histories. On the left portion the beginning of time histories are shown, in which only the response due the forced excitation is present, thus giving such a period of $T_{\Omega}=1 \mathrm{~s}$. The right portion of the figure shows the expanded time histories of the response due to the combined external and parametric excitations. It can be noticed in this case a complex dynamic behavior, in which there is periodic variations in the response's amplitude with a period $T_{r}=1 \mathrm{~s}$.

From the results shown in Fig. 16 the periodicity of the $(t>>0)$ system's response was equal to the period of the forced excitation, that is, for the above case $T_{\Omega}=T_{r}=1 \mathrm{~s}$. This result may suggest that the periodicity of the response for $t>>0$ is dominated by the frequency of forced excitation. However, before drawing this conclusion the last simulated case needs to be checked when $\Omega: \bar{\omega}_{n}=3: 20$.

Figure 17 shows the results obtained when $\Omega: \bar{\omega}_{n}=3: 20$. If, indeed the forced excitation was the responsible for dictating the period of the developed $(t>>0)$ system's response, the periodicity must be $T_{r}=0.33 \mathrm{~s}$, however the results point to a period equals to $T_{r}=1 \mathrm{~s}$ and consequently suggests a more complex process, that is, the period of the forced excitation is not the main factor that determines the period of the response.

The true rule that dictates which will be the periodicity of the developed system's response when $\Omega$ and $\omega_{n}$ are commensurable and $\lambda=2 \omega_{n}$ is given by the inverse of greatest common divisor of $\Omega$ and $\omega_{n}$, both given in Hertz. By applying this rule for the first case, that is, when $\Omega: \bar{\omega}_{n}=1: 20$ results: the greatest common divisor between $1 \mathrm{~Hz}$ and $20 \mathrm{~Hz}$ is $1 \mathrm{~Hz}$, in which the inverse is $1 \mathrm{~s}$. For the second case in which $\Omega: \bar{\omega}_{n}=3: 20$ result: the greatest common divisor between $3 \mathrm{~Hz}$ and $20 \mathrm{~Hz}$ is $1 \mathrm{~Hz}$, in which inverse is $1 \mathrm{~s}$. All these results are validates by the predictions shown in Figs 16 and 17.

\subsection{Case 3: $\Omega$ and $\omega_{n}$ incommensurable and $\lambda=2 \omega_{n}$}

In practical situations, unfortunately there are only few situations in which external and parametric excitations are commensurable. Consequently it is also of interest to investigate the case when that excitations are incommensurable. Although more common, the incommensurable case is much more complex when compared to the previous case. In 


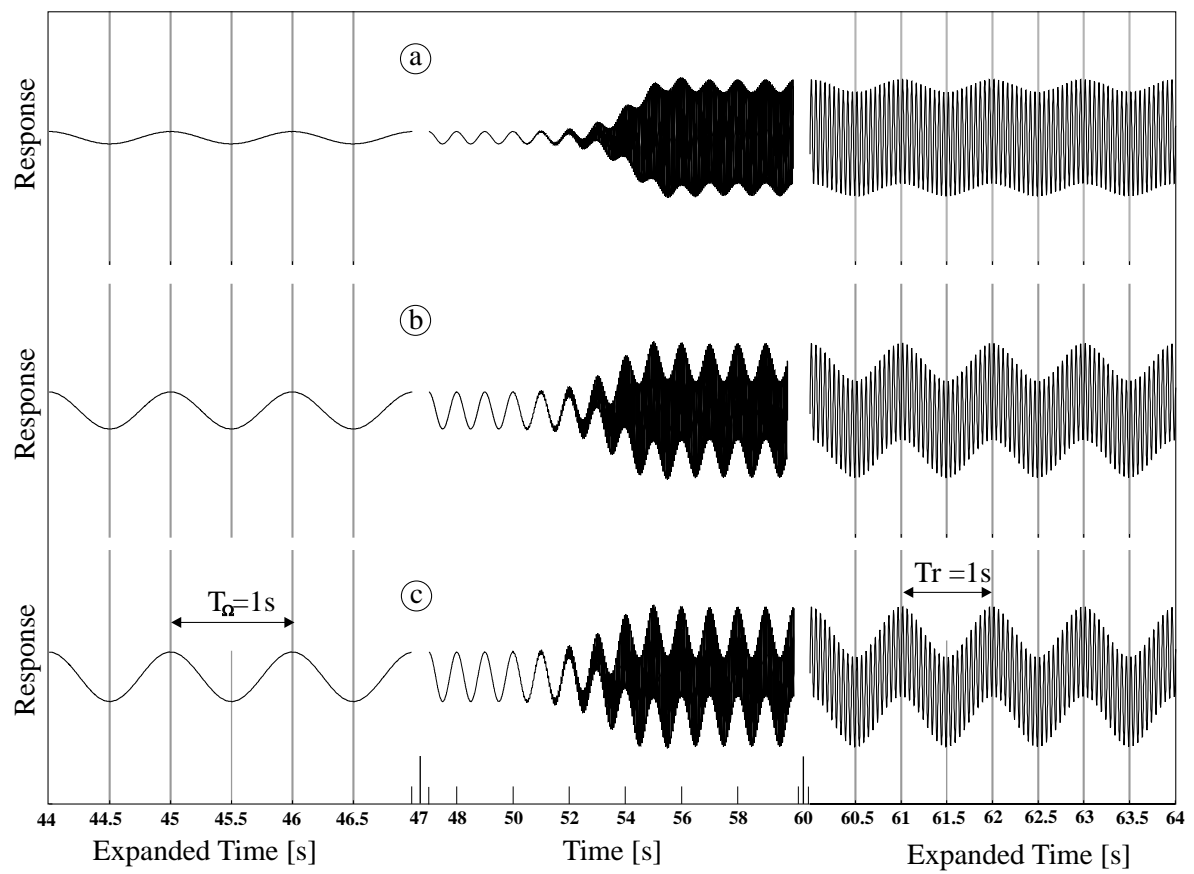

Fig. 16. Predicted system's dynamic behavior when $\Omega: \bar{\omega}_{n}=1: 20$ : (a) $H_{6}=0.47$; (b) $H_{6}=1.43$ and; (c) $H_{6}=1.90$.

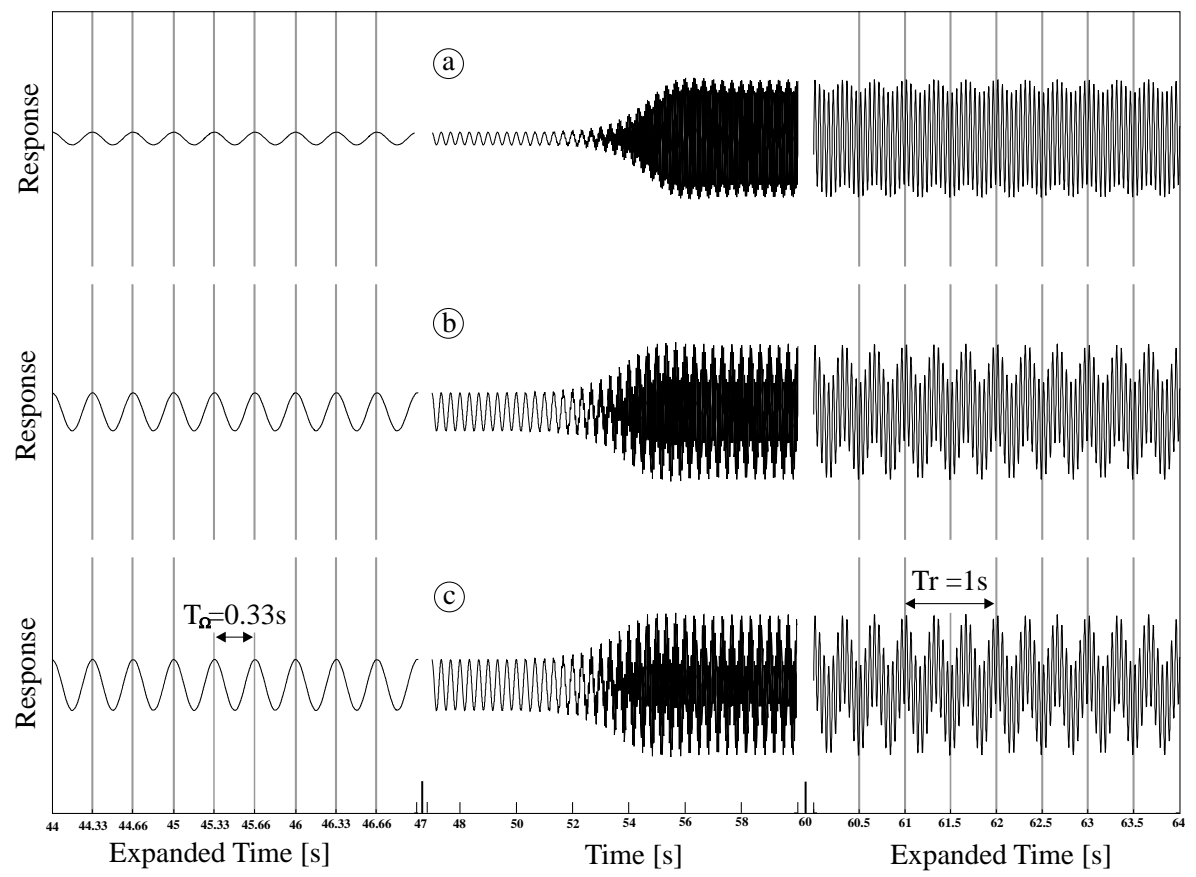

Fig. 17. Predicted system's dynamic behavior when $\Omega: \bar{\omega}_{n}=3: 20$ : (a) $H_{6}=0.47$; (b) $H_{6}=1.43$ and; (c) $H_{6}=1.90$.

addition, it was also observed that the rule shown in the earlier section no long is applicable when the excitation in question are incommensurable.

Several numerical simulations were performed in this case and the results are shown in Table 3 . The cases presented in this table show some specific ratios between $\Omega$ and $\omega_{n}$. Among these, it was simulated the cases of a 
Table 3

Result of several simulations when $\Omega$ and $\omega_{n}$ are incommensurable and $\lambda=2 \omega_{n}$

\begin{tabular}{ccc}
\hline Ratio $\Omega: \omega_{n}$ & Period [s] & Frequency $[\mathrm{Hz}]$ \\
\hline $1.25: 20$ & 0.80 & 1.25 \\
$1.33: 20$ & 0.75 & 1.33 \\
$1.50: 20$ & 2.00 & 0.50 \\
$1.66: 20$ & 0.60 & 1.66 \\
$1.75: 20$ & 4.00 & 0.25 \\
$3.25: 20$ & 4.00 & 0.25 \\
$3.33: 20$ & 0.30 & 3.33 \\
$3.50: 20$ & 2.00 & 0.50 \\
$3.66: 20$ & 3.00 & 0.33 \\
$3.75: 20$ & 0.80 & 1.25 \\
\hline
\end{tabular}

integer plus one half, a integer plus one third, a integer plus one quarter, etc. As results, it was found periodicity of the developed response for all special cases, even though no periodicity in the response was expected in the beginning.

Unfortunately from the results shown in Table 3 it was not possible to determine any rule that could be employed in order to predict the periodicity of the response. However, once and again the dynamic behavior has shown fantastic, and really complex behavior. Special attention must to be paid in future in order to better understand the cases when the excitations are incommensurable.

\section{Concluding remarks}

In the present investigation, a detailed theoretical and experimental study was performed on a flexible structure under the combined action of two different excitations, an external from a acoustic source and a parametric from a electrodynamic shaker.

The obtained theoretical and experimental results shown many interested aspects on the dynamics of the tested structure. When the excitation frequency are commensurable, the structure does not reach a steady-state regime, except in a very special condition when $\Omega=\omega_{n}$ and $\lambda=2 \omega n$. Still in the commensurable case, it was shown that there is a general rule that determine the periodicity of the structure's response. On the other hand, in the incommensurate case it does not obtained any rule that could be used to predict the periodicity of the response, even thought it was shown that exist a periodic motion for some special ratio between $\Omega$ and $\omega_{n}$ fixing $\lambda=2 \omega n$. In both cases, a fantastic and complex dynamic behavior was observed, showing the hard way to control they.

At the end, it also showed that all kind of exciting source have limitations and its own characteristics, which in turn, must to be known before they could be effectively used in experimental studies. For instance, if a certification does not had been made, probably some erroneous results using the acoustic source tuned about $18 \mathrm{~Hz}$ are present in the present investigation. Also due to the parametric certification, the effect of a small but present harmonic in the parametric excitation does not could be ignored and possible its effects will be attributed to the structure dynamic's behavior.

\section{Acknowledgments}

The authors gratefully acknowledge the support given to the reported reseach by the Conselho Nacional de Desenvolvimento Científico e Tecnológico, CNPq, Brazil. The Laboratory support received from University of S ão Paulo is greatly appreciated.

\section{References}

[1] T.J. Anderson, A.H. Nayfeh and B. Balachandran, Experimental verification of the importance of the nonlinear curvature in the response of a cantilever beam, Journal of Vibration and Acoustics 118 (1996), 21-27. 
[2] A.P. Boresi, R.J. Schimidt and O.M. Sidebottom, Advanced mechanics of materials, New York: Wiley \& Sons, 1993.

[3] J.R. Callinan, S.C. Galea and S. Sanderson, Finite element analysis of bonded repairs to edge cracks in panels subjected to acoustic excitation, Composite Structures 38(1-4) (1997), 649-660.

[4] M. CartmelL, Introduction to linear, parametric and nonlinear vibration, London: Chapman and Hall, 1990.

[5] M.P. Cartmell, The equations of motion for a parametrically excited cantilever beam, Journal of Sound and Vibration 143(3) (1990), $395-406$.

[6] R.M. Evan-iwanowski, On the parametric response of structures, Applied Mechanics Reviews 18(9) (1965), 699-702.

[7] F. Fahy, Sound and Structural Vibration, London: Academic Press, 1987.

[8] D.I.M. Forehand and M.P. Cartmell, On the derivation of the equations of motion for a parametrically excited cantilever beam, Journal of Sound and Vibration 245(1) (2001), 165-177.

[9] D. Gomes da silva and P.S. Varoto, On the Suffiency of Classical Response Models in Predicting the Dynamic Behavior of Flexible Structures, In: Proceeding of the Twenty Second International Modal Analysis Conference. Dearborn, MI, USA, 2004, 21.

[10] N. Haquang and D.T. Mook, A non-linear analysis of the interactions between parametric and external excitations, Journal of Sound and Vibration 118(3) (1987), 425-439.

[11] N. Haquang and D.T. Mook, Non-linear structural vibration under combined parametric and external excitations, Journal of Sound and Vibration 118(2) (1987), 291-306.

[12] L. Meirovitch, Methods of analytical dynamics, New York: McGraw-Hill, 1970.

[13] A.H. Nayfeh, Nonlinear Interactions: Analytical, Computational, and Experimental Methods, New York: Wiley \& Sons, 2000.

[14] A.H. Nayfeh and D.T. Mook, Nonlinear oscillations, New York: Wiley \& Sons, 1979.

[15] S.A. Nayfeh, A.H. Nayfeh and D.T. Mook, Nonlinear response of a taut string to longitudinal and transverse end excitation, Journal of Vibration and Control 1 (1995), 307-334.

[16] G.R. Tomlinson, Force distortion in resonance testing of structures with electro-dynamic vibration exciters, Journal of Sound and Vibration 63(3) (1979), 337-350.

[17] P.S. Varoto and L.P.R. Oliveira, On the force drop off phenomenon in shaker testing in experimental modal analysis, Shock and Vibration 9 (2002), 165-175.

[18] L.D. Zavodney and A.H. Nayfeh, The non-linear response of a slender beam carrying a lumped mass to a principal parametric excitation: theory and experiment, International Journal of Non-linear Mechanics 24(2) (1989), 105-125. 

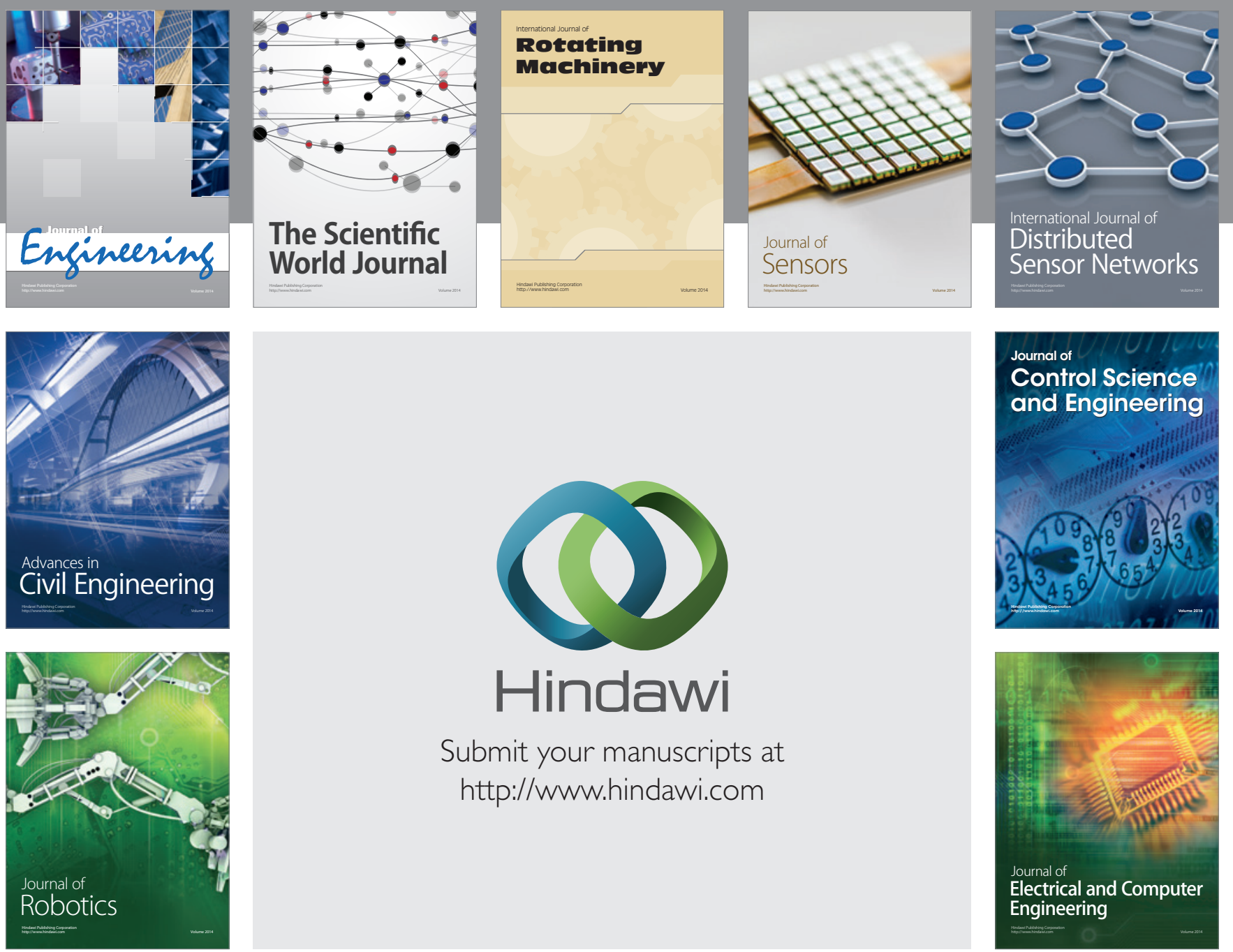

Submit your manuscripts at

http://www.hindawi.com
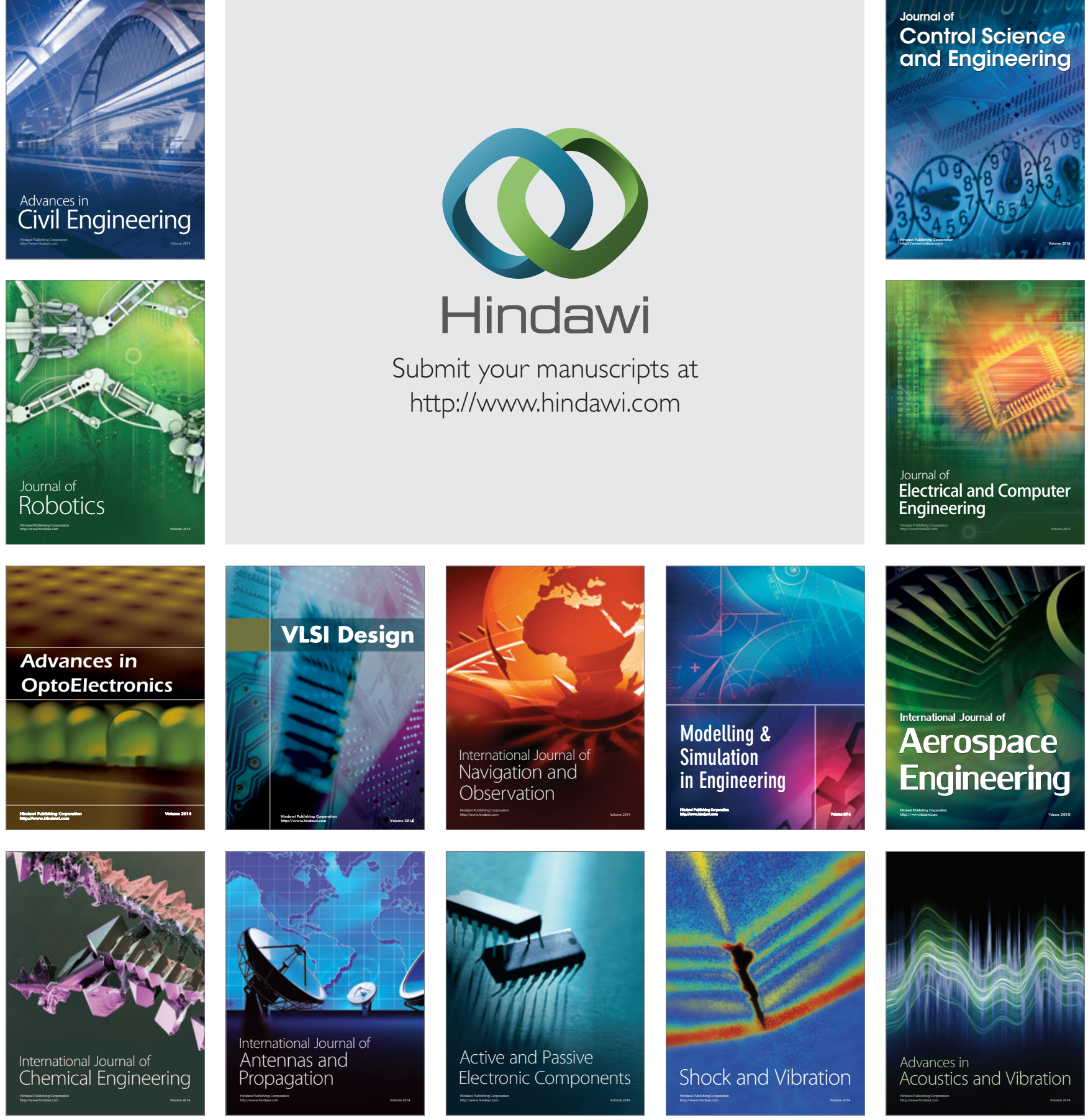